\title{
Stability analysis of five-grade Leishmania epidemic model with harmonic mean-type incidence rate
}

\author{
Karim Khan', Rahat Zarin², Amir Khan ${ }^{3}$ Abdullahi Yusuf ${ }^{4,5^{*}}$ (D, Mohammed Al-Shomrani ${ }^{6 *}$ and Arif Ullah
}

\section{"Correspondence:}

yusufabdullahi@fud.edu.ng;

malshamrani@kau.edu.sa

${ }^{4}$ Department of Computer

Engineering, Biruni University, Istanbul, Turkey

${ }^{6}$ Department of Mathematics, Faculty of Science, King Abdulaziz University, Jeddah 21589, Saudi Arabia

Full list of author information is available at the end of the article

\section{Springer}

\begin{abstract}
In this paper, we discuss the Anthroponotic Cutaneous Leishmania transmission. In addition, we develop a mathematical model for the Anthroponotic Cutaneous Leishmania transmission and consider its qualitative behavior. We derive the threshold number $R_{0}$ of the model using the next generation method. In the disease-free case, we carry out the local and global stability under the condition $R_{0}<1$. Moreover, we derive the global stability at the disease-free equilibrium point by utilizing the Castillo-Chavez method. On the other hand, at the endemic equilibrium point, we show the local and global stability to be held under specific conditions and $R_{0}>1$. We also establish the global stability at the endemic equilibrium point with the help of a geometrical approach, which is a generalization of Lyapunov theory, by using a second additive compound matrix. Finally, we take into account the sensitivity analysis of the threshold number with other parameters. We also discuss several graphs of important parameters.
\end{abstract}

Keywords: Compound matrix; Sensitivity analysis; Geometric approach; Stability analysis

\section{Introduction}

Leishmaniasis is the sixth most common disease affecting public health worldwide. It is a vector borne disease transmitted among mammalian hosts by the female sand fly. Most common animals carrying the Leishmania parasite are rodents, dogs, cattles, and foxes, this parasite primarily affecting the skin. Leishmaniasis cannot be transmitted from person to person through touch, blood transfusion, congenitally from mother to baby, infected needles and syringes, and so on. Different lab tests are performed to diagnose this parasite; for example, blood test for antibodies against the parasite, urine tests, and polymerase chain reaction (PCR) test can identify parasite based on its genetic fingerprint, and smear test identifies the parasite through a microscope.

The transmission of parasites is carried out by the species of genera Phelobotomus type. The sand flies are the main transmitter of these parasites. The habitat of these flies enjoys a wide range, from desert to tropical rain forest. Not only these but also several hosts in which dogs, chickens, humans, mammals, livestock, and vertebrates are considered to be

(c) The Author(s) 2021. This article is licensed under a Creative Commons Attribution 4.0 International License, which permits use sharing, adaptation, distribution and reproduction in any medium or format, as long as you give appropriate credit to the original author(s) and the source, provide a link to the Creative Commons licence, and indicate if changes were made. The images or other third party material in this article are included in the article's Creative Commons licence, unless indicated otherwise in a credit line to the material. If material is not included in the article's Creative Commons licence and your intended use is not permitted by statutory regulation or exceeds the permitted use, you will need to obtain permission directly from the copyright holder. To view a copy of this licence, visit http://creativecommons.org/licenses/by/4.0/ 
the main hosts [1]. The color of these sand flies is sandy, and normally they are $2-3-\mathrm{mm}$ long. The latent period of this sand fly is considered to be between three and seven days [2]. From the ground level, the sandflies attain $2.51 \mathrm{~m}(8.3 \mathrm{ft})$ as their maximum height [3]. The incidental transference risk of blood-borne pathogens of humans is reduced by the use of animal blood and is considered to be cheaper than the conservation of animals and their preparation for feeding sandfly [4]. The fecundity of species is badly affected by the extreme temperatures [5]. Some recent studies on Cutaneous Leishmaniasis epidemic models are described in [6-12]. Mathematical modeling is one of powerful tools to describe the dynamical behavior of different diseases [13-18].

Grouping and grading: Computational modeling of Leishmaniasis is developed to verify the prevalence and clearance of diseases in an endemic region. Patients are divided into two group's based on the disease grading. Group one includes patients from grades I-III, and group two includes grades IV-V. Grading of CL lesions is categorized on the basis of their clinical presentations. Specifically, the size (i.e., mean diameter) and shape of each CL lesion are assessed and subsequently used to classify the lesions on a numeric scale, from grade 1 (i.e., papule with mild overlying fine scales of size $\leq 1 \mathrm{~cm}$ ), grade $2(\leq 1 \mathrm{~cm})$ nodule formation, grade $3(<2 \mathrm{~cm})$ vesicle formation or ulceration, grade $4(2-4 \mathrm{~cm})$ vesicle formation and ulceration with rolled edges, and grade $5(>4 \mathrm{~cm})$ vesicle formation, ulceration, and superadded infection.

\subsection{Types of leishmaniasis}

I. Mucocutaneous Leishmaniasis (MCL) is a less common form of Leishmaniasis, which is the partial or complete destruction of mucous membranes in the nose, throat, or mouth. It is either CL skin lesion to mucosa membrane or carried by direct bite of a sand fly on mucus membranes.

II. Visceral Leishmaniasis (VL) is the most lethal disease, which appears from days to year after a bite of an infected sand fly. This disease affects the internal organ like the liver, spleen, and bone marrow. Patients are symptomatically present with enlargement of the spleen and liver, weight loss, and episodes of fever. In Pakistan, both MCL and VL are rare.

III. Cutaneous Leishmaniasis (CL) is an ulcer appearance on the skin, which is usually developed in a couple of days after a bite of the sand fly. If the immunity of the patient is strong enough, then mostly these sores may heal spontaneously within a few days. According to the World Health Organization (WHO), 1-1.5 million new cases of CL are reported every year worldwide. Pakistan is one of the countries where the incident of CL is increasing yearly, and its main reason is poverty, negligible planning for vector control, inadequate medical facilities, armed conflicts, and mass migration of humans and cattle.

\subsection{Treatment methods}

Standard drugs: Meglumine antimoniate and sodium stibogluconate are used as a first-line conventional treatment worldwide to treat $\mathrm{CL}$. These drugs are used oral and injectable. Besides their hard administration, it offers many side effects, and drugs are costly as well.

Conventional treatment: Some conventional domestic methods help in healing in $\mathrm{CL}$ wound: applying garlic water on the lesion, cleaning wound repeatedly with antiseptics, and placing a hot stone on wound have been reported by people.

Photodynamic therapy: Photodynamic therapy (PDT), the use of light of specific wavelength and energy, is a rapidly evolving therapeutic option for the treatment of CL. The 
FDA has approved indications of PDT for various skin diseases, actinic keratosis, basal cell carcinoma, superficial squamous cell carcinoma, psoriasis, acne vulgaris, and sarcoidosis, verruca Vulgaris, and condyloma acuminatum. Treatment protocols of PDT are easy, patients' comfort and lesion response to PDT make this the best option to treat CL.

Treatment protocols: Herein we present a number of cases of CL lesions at different sites of the body treated with PDT from Pakistan. These lesions had been diagnosed clinically as cutaneous Leishmaniasis (CL). The patients had received treatment (i.e., pentavalent antimoniate) with no satisfactory outcome. Clinical assessment showed that the patients had developed resistance to pentavalent antimoniate. The patients were planned for photodynamic therapy (PDT). Written and informed consent was obtained from the patient. To perform PDT, the lesions and the adjacent skin were cleaned and scrubbed to remove the necrotic layer and exudates dried up. The photosensitizer, methyl aminolevulinate (MAL), was applied locally on the lesions under an adhesive covering, followed by an incubation period of 3 hours for the absorption of the photosensitizer. After the incubation period, the cream was removed, and the lesion was washed with normal saline. The lesion was irradiated with red laser light (wavelength $635 \mathrm{~nm}$ ); a light energy dose of $75 \mathrm{~J} / \mathrm{cm}^{2}$ was delivered as per the institutional protocol. The patient received three sessions of PDT; the first two sessions were repeated at two weeks intervals, whereas the third session was given at one-month intervals. The patient showed a complete response to PDT, being followed up regularly, and has remained disease-free for over two years.

\section{Model formulation}

Mathematical modeling of epidemic diseases has been widely studied by researchers [1921]. The model is a modified version of Khan et al. [22], in which the total population $N(t)$ is partitioned into the following subpopulations. These classes are classified into four human population subclasses $S_{h}(t), E_{h}(t), I_{1 h}(t), I_{2 h}(t)$, and $R_{h}(t)$, denoting the susceptible, exposed, infected people from grades I-III, infected people in grades IV and $\mathrm{V}$, and recovered people. The three vector population subclasses $S_{v}(t), E_{v}(t)$, and $I_{v}(t)$ represent susceptible, exposed, and infected vectors; the total human population is represented by $N_{h}(t)=S_{h}(t)+E_{h}(t)+I_{1 h}(t)+I_{2 h}(t)+R_{h}(t)$. Total total vector population is $N_{\nu}(t)=S_{v}(t)+E_{v}(t)+I_{\nu}(t)$.

The model is given as follows:

$$
\left\{\begin{array}{l}
\frac{d S_{h}}{d t}=\Gamma_{h}-\frac{2 a b I_{v}(t) S_{h}(t)}{S_{h}(t)+I_{v}(t)}-\mu_{h} S_{h}(t), \\
\frac{d E_{h}}{d t}=\frac{2 a b I_{\nu}(t) S_{h}(t)}{S_{h}(t)+I_{v}(t)}-\left(k_{1}+\theta+\mu_{h}\right) E_{h}(t), \\
\frac{d I_{1 h}}{d t}=k_{1} E_{h}(t)-\left(\beta+\mu_{h}+k_{2}\right) I_{1 h}(t), \\
\frac{d I_{2 h}}{d t}=k_{2} I_{1 h}(t)-\left(\mu_{h}+\theta_{1}\right) I_{2 h}(t), \\
\frac{d R_{h}}{d t}=\theta E_{h}(t)+\beta I_{h}(t)+\theta_{1} I_{2 h}(t)-\mu_{h} R_{h}(t), \\
\frac{d S_{v}}{d t}=\Gamma_{v}-a c I_{1 h}(t) S_{v}(t)-a d I_{2 h}(t) S_{v}(t)-\mu_{v} S_{v}(t), \\
\frac{d E_{v}}{d t}=a c I_{1 h}(t) S_{v}(t)+a d I_{2 h}(t) S_{v}(t)-\left(\mu_{v}+k_{3}\right) E_{v}(t), \\
\frac{d I_{v}}{d t}=k_{3} E_{v}(t)-\mu_{v} I_{v}(t),
\end{array}\right.
$$

with

$$
S_{h}(t), E_{h}(t), I_{1 h}(t), I_{2 h}(t), R_{h}(t), S_{v}(t), E_{v}(t), I_{v}(t) \geq 0 .
$$




\subsection{Basic properties of the model}

The total-population dynamics is represented by

$$
\begin{aligned}
& \frac{d N_{h}(t)}{d t}=\Gamma_{h}-\mu_{h} N_{h}(t), \\
& \frac{d N_{v}(t)}{d t}=\Gamma_{v}-\mu_{v} N_{v}(t) .
\end{aligned}
$$

The feasible region (biological) $\Delta$ is

$$
\Delta=\left\{\left(S_{h}, E_{h}, I_{1 h}, I_{2 h}, R_{h}, S_{v}, E_{v}, I_{v}\right) \in \mathbb{R}_{+}^{\not}, N_{v} \leq \frac{\Gamma_{v}}{\mu_{v}} ; N_{h} \leq \frac{\Gamma_{h}}{\mu_{h}}\right\} .
$$

From Eqs. (2) and (3) we obtain

$$
N_{h} \rightarrow \frac{\Gamma_{h}}{\mu_{h}}, \quad N_{v} \rightarrow \frac{\Gamma_{v}}{\mu_{v}} \quad \text { as } t \rightarrow \infty
$$

which shows that the model is well posed and $\Delta$ is a positively invariant domain.

Lemma 1 The orthant $R_{+}^{7}$ is invariant positively for system (1).

Proof Let $X=\left(S_{h}, E_{h}, I_{1 h}, I_{2 h}, R_{h}, S_{v}, E_{v}, I_{v},\right)^{T}$ and assume that $a_{11}=\mu_{h}+\frac{2 a b\left(I_{v}\right)^{2}}{\left(S_{h}+I_{v}\right)^{2}}, a_{21}=$ $\frac{2 a b I_{v}^{2}}{\left(S_{h}+I_{v}\right)^{2}}, a_{22}=\left(k_{1}+\theta+\mu_{h}\right), a_{18}=\frac{-2 a b\left(S_{h}\right)^{2}}{\left(S_{h}+I_{v}\right)^{2}}, a_{28}=\frac{-2 a b\left(S_{h}\right)^{2}}{\left(S_{h}+I_{v}\right)^{2}}, a_{66}=a c I_{1 h}+a d I_{2 h}+\mu_{v}$, and $a_{76}=a c I_{1 h}+a d I_{2 h}$.

System (1) is expressed in the form

$$
\frac{d X}{d t}=L X+B
$$

where

$$
\begin{aligned}
L & =\left(\begin{array}{cccccccc}
-a_{11} & 0 & 0 & 0 & 0 & 0 & 0 & a_{18} \\
a_{21} & -a_{22} & 0 & 0 & 0 & 0 & 0 & a_{28} \\
0 & k_{1} & -\left(\beta+k_{2}+\mu_{h}\right) & 0 & 0 & 0 & 0 & 0 \\
0 & 0 & k_{2} & -\left(\theta_{1}+\mu_{h}\right) & 0 & 0 & 0 & 0 \\
0 & \theta & \beta & \theta_{1} & -\mu_{h} & 0 & 0 & 0 \\
0 & 0 & -a c S_{v} & -a d S_{v} & 0 & -a_{66} & 0 & 0 \\
0 & 0 & a c S_{v} & a d S_{v} & 0 & a_{76} & -\left(\mu_{v}+k_{2}\right) & 0 \\
0 & 0 & 0 & 0 & 0 & k_{3} & -\mu_{v}
\end{array}\right) \\
B & \left(\begin{array}{c}
2 a b S_{h}-2 a b S_{h} \\
0 \\
0 \\
0 \\
2 a c S_{v}-2 a c S_{v} \\
0 \\
0
\end{array}\right)
\end{aligned}
$$


Here we see that $L$ is the Metzler matrix as it has nonnegative entries on its of-diagonal and $B \geq 0$. Hence we conclude that system (1) is positively invariant in $R_{+}^{8}$.

Lemma 2 If solutions of system (1) exist, then they are positive under the initial conditions (2) for all $t>0$.

Proof Let us assume that the solutions exist in $I$ for all $t \in I \subset[0, \infty)$. Consider the second equation of (1). Its solution has the following form:

$$
\begin{aligned}
E_{h}(t)= & E_{h}(0) \exp \left\{-\left(k_{1}+\theta+\mu_{h}\right) t\right\}+\exp \left\{-\left(k_{1}+\theta+\mu_{h}\right) t\right\} \\
& \times \int_{0}^{t} \frac{2 a b I_{v}(x) S_{h}(x)}{S_{h}(x)+I_{v}(x)} \exp \left\{\left(k_{1}+\theta+\mu_{h}\right) x\right\} d x .
\end{aligned}
$$

We also take the third equation, and its solution has the following form:

$$
\begin{aligned}
I_{1 h}(t)= & I_{1 h}(0) \exp \left\{-\left(\beta+k_{2}+\mu_{h}\right) t\right\}+\exp \left\{-\left(\beta+k_{2}+\mu_{h}\right) t\right\} \\
& \times \int_{0}^{t} k_{1} E_{h}(y) \exp \left\{\left(\beta+k_{2}+\mu_{h}\right) y\right\} d y .
\end{aligned}
$$

We can clearly see from the above solutions that these are strictly positive. In the same fashion, we can show that $S_{h}, R_{h}, S_{v}, E_{v}, I_{2 h}$, and $I_{v}$ possess nonnegative solutions.

\subsection{Basic reproductive number $\boldsymbol{R}_{0}$}

The disease-free equilibrium point of system (1) is

$$
E^{0}=\left(S_{h}^{0}, E_{h}^{0}, I_{1 h}^{0}, I_{2 h}^{0}, R_{h}^{0}, S_{v}^{0}, E_{v}^{0}, I_{v}^{0}\right)=\left(\frac{\Gamma_{h}}{\mu_{h}}, 0,0,0,0, \frac{\Gamma_{v}}{\mu_{v}}, 0,0\right) .
$$

Let $\left(E_{h}, I_{1 h}, I_{2 h}, E_{v}, I_{v}\right)$ be our infected compartment. Then from system (1) we have:

$$
\left\{\begin{array}{l}
\frac{d E_{h}}{d t}=\frac{2 a b I_{v}(t) S_{h}(t)}{S_{h}(t)+I_{v}(t)}-\left(k_{1}+\theta+\mu_{h}\right) E_{h}(t), \\
\frac{d I_{1 h}}{d t}=k_{1} E_{h}(t)-\left(\beta+\mu_{h}+k_{2}\right) I_{1 h}(t), \\
\frac{d I_{2 h}}{d t}=k_{2} I_{1 h}(t)-\left(\mu_{h}+\theta_{1}\right) I_{2 h}(t), \\
\frac{d E_{v}}{d t}=a c I_{1 h}(t) S_{v}(t)+a d I_{2 h}(t) S_{v}(t)-\left(\mu_{v}+k_{3}\right) E_{v}(t), \\
\frac{d I_{v}}{d t}=k_{3} E_{v}(t)-\mu_{v} I_{v}(t) .
\end{array}\right.
$$

Using the next generation matrix approach [23, 24], the Jacobian matrix $J$ for this system at the disease-free equilibrium point is given by

$$
J\left(E^{0}\right)=\left(\begin{array}{ccccc}
-\left(k_{1}+\theta+\mu_{h}\right) & 0 & 0 & 0 & 2 a b \\
k_{1} & -\left(\beta+k_{2}+\mu_{h}\right) & 0 & 0 & 0 \\
0 & k_{2} & -\left(\mu_{h}+\theta_{1}\right) & 0 & 0 \\
0 & \frac{a c \Gamma_{v}}{\mu_{v}} & \frac{a d \Gamma_{v}}{\mu_{v}} & -\left(\mu_{v}+k_{3}\right) & 0 \\
0 & 0 & 0 & k_{3} & -\mu_{v}
\end{array}\right) .
$$


Now decomposing the matrix $J$ in terms of $F$ and $V$ as $J=F-V$, we get

$$
\begin{aligned}
F & =\left(\begin{array}{ccccc}
0 & 0 & 0 & 0 & 2 a b \\
0 & 0 & 0 & 0 & 0 \\
0 & 0 & 0 & 0 & 0 \\
0 & \frac{a c \Gamma_{v}}{\mu_{v}} & \frac{a d \Gamma_{v}}{\mu_{v}} & 0 & 0 \\
0 & 0 & 0 & 0 & 0
\end{array}\right), \\
-V & =\left(\begin{array}{ccccc}
\left(k_{1}+\theta+\mu_{h}\right) & 0 & 0 & 0 & 0 \\
-k_{1} & \left(\beta+k_{2}+\mu_{h}\right) & 0 & 0 & 0 \\
0 & -k_{2} & \left(\mu_{h}+\theta_{1}\right) & 0 & 0 \\
0 & 0 & 0 & \left(\mu_{v}+k_{3}\right) & 0 \\
0 & 0 & 0 & -k_{3} & \mu_{v}
\end{array}\right),
\end{aligned}
$$

and

$$
-V^{-1}=\left(\begin{array}{ccccc}
\frac{1}{\left(k_{1}+\theta+\mu_{h}\right)} & 0 & 0 & 0 & 0 \\
\frac{k_{2}}{\left(\beta+k_{2}+\mu_{h}\right)\left(k_{1}+\theta+\mu_{h}\right)} & \frac{1}{\left(\beta+k_{2}+\mu_{h}\right)} & 0 & 0 & 0 \\
\frac{k_{2}^{2}}{\left(\beta+k_{2}+\mu_{h}\right)\left(\theta+k_{1}+\mu_{h}\right)\left(\mu_{h}+\theta_{1}\right)} & \frac{k_{2}}{\left(\mu_{h}+\theta_{1}\right)\left(K_{2}+\beta+\mu_{h}\right)} & \frac{1}{\mu_{h}+\theta_{1}} & 0 & 0 \\
0 & 0 & 0 & \frac{1}{\left(\mu_{v}+k_{3}\right)} & 0 \\
0 & 0 & 0 & \frac{1}{k_{3} \mu_{v}+\mu_{v}^{2}} & \frac{1}{\mu_{v}}
\end{array}\right) .
$$

Now

$$
-F V^{-1}=\left(\begin{array}{ccccc}
0 & 0 & 0 & \frac{2 a b}{\mu_{v}\left(\mu_{v}+k_{3}\right)} & \frac{2 a b}{\mu_{v}} \\
0 & 0 & 0 & 0 & 0 \\
0 & 0 & 0 & 0 & 0 \\
\bar{A} & \bar{B} & \frac{a d \Gamma_{v}}{\mu_{v}\left(\mu_{h}+\theta_{1}\right)} & 0 & 0 \\
0 & 0 & 0 & 0 & 0
\end{array}\right),
$$

where

$$
\left\{\begin{array}{l}
\bar{A}=\frac{a c \Gamma_{v} k_{2}}{\mu_{v}\left(\beta+k_{2}+\mu_{h}\right)\left(k_{1}+\theta+\mu_{h}\right)}+\frac{a d \Gamma_{v} k_{2}^{2}}{\mu_{v}\left(\beta+k_{2}+\mu_{h}\right)\left(\theta+k_{1}+\mu_{h}\right)\left(\mu_{h}+\theta_{1}\right)}, \\
\bar{B}=\frac{a c \Gamma_{v}}{\mu_{v}\left(\beta+k_{2}+\mu_{h}\right)}+\frac{a d \Gamma_{v} k_{2}}{\mu_{v}\left(\mu_{h}+\theta_{1}\right)\left(\beta+k_{2}+\mu_{h}\right)} .
\end{array}\right.
$$

The characteristics equation of $\left[-F V^{-1}\right]$ becomes

$$
-\lambda^{3}\left(\lambda^{2}-\bar{A} \frac{2 a b}{\mu_{\nu}\left(k_{3}+\mu_{v}\right)}\right)=0 .
$$

The dominant eigenvalue gives us $R_{0}$, that is,

$$
\text { basic reproduction number }=\sqrt{\left(\frac{2 a^{2} b \Gamma_{v} k_{2}\left(c\left(\mu_{h}+\theta_{1}\right)+d k_{2}\right)}{\mu_{v}^{2}\left(\beta+k_{2}+\mu_{h}\right)\left(k_{1}+\theta+\mu_{h}\right)\left(\mu_{v}+k_{3}\right)\left(\mu_{h}+\theta_{1}\right)}\right)} .
$$

\section{Local stability}

In this section, we establish the local stability of system (1) in this section at the diseasefree point $E^{0}$ and endemic equilibrium point $E^{*}$. 


\subsection{Geometric interpretation of stability}

For the DFE, the local stability implies that if there is a small perturbation of the system (e.g., a small number of infected individuals is introduced into the population), then after some time, the system will return to the DFE. However, a big perturbation may be able to drive the system to a different behavior, for example, convergence to the EE. If the stability of the DFE is global, then no matter the size of the perturbation, the disease will not be able to persist in the population.

On the other hand, the local stability of an equilibrium point means that if you put the system somewhere nearby the point, then it will move itself to the equilibrium point in some time. The global stability means that the system will come to the equilibrium point from any possible starting point (i.e., there is no "nearby" condition). Even in more physical interpretation, it could be like this: If DFE or EE is locally stable, then all epidemiological situations not-so-much different from the given stable equilibrium will (with time) evolve to (or transform into) the equilibrium point. Also, this means that the equilibria are stable to small perturbations: if you push the situation a bit out from the equilibrium point, then the situation will return back on its own (from the physicist's point of view, this means that the equilibrium may be a stable situation in real life because the real world always is somewhat noisy). The global stability of an equilibrium point in this case maybe described as "the inevitable fate of the epidemic process regardless of its starting situation". However, a caveat should be put that this "inevitability" holds as long as the world strictly follows the underlying mathematical model of the epidemic process. For still even more lay (or medical) audience, all these things may be easily interpreted in terms of "adding or removing a small number of infectious people", but we believe it would make the central idea less clear.

\subsection{At disease free equilibrium point}

Theorem 3.1 System (1) at disease-free equilibrium point $E^{0}$ is locally asymptotically stable if $R_{0}<1$.

Proof For system (1), the Jacobian matrix at $E^{0}$ is given by

$$
J^{|0|}=\left(\begin{array}{cccccccc}
-\mu_{h} & 0 & 0 & 0 & 0 & 0 & 0 & -2 a b \\
0 & -\left(k_{1}+\theta+\mu_{h}\right) & 0 & 0 & 0 & 0 & 0 & 2 a b \\
0 & k_{1} & -\left(\beta+k_{2}+\mu_{h}\right) & 0 & 0 & 0 & 0 & 0 \\
0 & 0 & k_{2} & -\left(\mu_{h}+\theta_{1}\right) & 0 & 0 & 0 & 0 \\
0 & 0 & \beta & \theta_{1} & -\mu_{h} & 0 & 0 & 0 \\
0 & 0 & -\frac{a c \Gamma_{v}}{a \mu_{v}} & -\frac{a d \Gamma_{v}}{\mu_{v}} & 0 & -\mu_{v} & 0 & 0 \\
0 & 0 & \frac{a c \Gamma_{v}}{\mu_{\nu}} & \frac{a d \Gamma_{v}}{\mu_{v}} & 0 & 0 & -\left(\mu_{v}+k_{2}\right) & 0 \\
0 & 0 & 0 & 0 & 0 & 0 & k_{3} & -\mu_{v}
\end{array}\right) .
$$

We can clearly see that three eigenvalues are negative, namely, $\lambda_{1}=-\mu_{h}, \lambda_{2}=-\mu_{h}$, and $\lambda_{3}=-\mu_{\nu}$. For the rest of eigenvalues, we consider the following reduced matrix:

$$
J_{1}^{|0|}=\left(\begin{array}{ccccc}
-\left(k_{1}+\theta+\mu_{h}\right) & 0 & 0 & 0 & 2 a b \\
k_{1} & -\left(\beta+k_{2}+\mu_{h}\right) & 0 & 0 & 0 \\
0 & k_{2} & -\left(\mu_{h}+\theta_{1}\right) & 0 & 0 \\
0 & \frac{a c \Gamma_{v}}{\mu_{v}} & \frac{a d \Gamma_{v}}{\mu_{v}} & -\left(\mu_{v}+k_{2}\right) & 0 \\
0 & 0 & 0 & k_{3} & -\mu_{v}
\end{array}\right) .
$$


After simplification, we get:

$$
\begin{aligned}
J_{2}^{|0|}= & \left(\begin{array}{ccccc}
-L_{11} & 0 & 0 & 0 & 2 a b \\
0 & -L_{22} & 0 & 0 & 2 a b k_{1} \\
0 & 0 & -L_{33} & 0 & 2 a b k_{1} k_{2} \\
0 & 0 & 0 & -L_{44} & L_{45} \\
0 & 0 & 0 & 0 & -L_{55}
\end{array}\right), \\
L_{11}= & \left(k_{1}+\theta+\mu_{h}\right), \quad L_{22}=\left(k_{1}+\theta+\mu_{h}\right)\left(k_{2}+\beta+\mu_{h}\right), \\
L_{33}= & \left(k_{1}+\theta+\mu_{h}\right)\left(k_{2}+\beta+\mu_{h}\right)\left(\theta_{1}+\mu_{h}\right), \\
L_{44}= & \left(k_{1}+\theta+\mu_{h}\right)^{2}\left(k_{2}+\beta+\mu_{h}\right)^{2}\left(\theta_{1}+\mu_{h}\right)\left(\mu_{v}+k_{2}\right), \\
L_{55}= & \frac{2 a b k_{1} k_{2} k_{3} a d \Gamma_{v}}{\mu_{v}}\left(k_{1}+\theta+\mu_{h}\right)\left(k_{2}+\beta+\mu_{h}\right) \\
& +\frac{2 a b k_{1} k_{2} a c \Gamma_{v}}{\mu_{v}} L_{33}-\mu_{v}\left(\mu_{v}+k_{2}\right) L_{33} L_{22}, \\
L_{45}= & \frac{2 a b k_{1} k_{2} a d \Gamma_{v}}{\mu_{v}}\left(k_{1}+\theta+\mu_{h}\right)\left(k_{2}+\beta+\mu_{h}\right)+\frac{2 a b k_{1} a c \Gamma_{v}}{\mu_{v}} L_{33} .
\end{aligned}
$$

The eigenvalues of $J_{2}^{|0|}$ take the following form:

$$
\begin{aligned}
& \lambda_{4}=-\left(k_{1}+\theta+\mu_{h}\right)<0, \quad \lambda_{5}=-\left(k_{1}+\theta+\mu_{h}\right)\left(k_{2}+\beta+\mu_{h}\right)<0, \\
& \lambda_{6}=-\left(k_{1}+\theta+\mu_{h}\right)\left(k_{2}+\beta+\mu_{h}\right)\left(\theta_{1}+\mu_{h}\right)<0, \\
& \lambda_{7}=-\left(k_{1}+\theta+\mu_{h}\right)^{2}\left(k_{2}+\beta+\mu_{h}\right)^{2}\left(\theta_{1}+\mu_{h}\right)\left(\mu_{v}+k_{2}\right)<0,
\end{aligned}
$$

and

$$
\begin{aligned}
\lambda_{8}= & \frac{2 a b k_{1} k_{2} k_{2} a d \Gamma_{v}}{\mu_{v}}\left(k_{1}+\theta+\mu_{h}\right)\left(k_{2}+\beta+\mu_{h}\right) \\
& +\frac{2 a b k_{1} k_{2} a c \Gamma_{v}}{\mu_{v}}\left(k_{1}+\theta+\mu_{h}\right)\left(k_{2}+\beta+\mu_{h}\right)\left(\theta_{1}+\mu_{h}\right) \\
& -\mu_{v}\left(\mu_{v}+k_{2}\right)\left(k_{1}+\theta+\mu_{h}\right)^{2}\left(k_{2}+\beta+\mu_{h}\right)^{2}\left(\theta_{1}+\mu_{h}\right)<0 .
\end{aligned}
$$

The last eigenvalue also has the negative real part since

$$
\left(\frac{2 a^{2} b \Gamma_{v} k_{2}\left(c\left(\mu_{h}+\theta_{1}\right)+d k_{2}\right)}{\mu_{v}^{2}\left(\beta+k_{2}+\mu_{h}\right)\left(k_{1}+\theta+\mu_{h}\right)\left(\mu_{v}+k_{3}\right)\left(\mu_{h}+\theta_{1}\right)}\right)<1
$$

implies that

$$
\sqrt{\left(\frac{2 a^{2} b \Gamma_{v} k_{2}\left(c\left(\mu_{h}+\theta_{1}\right)+d k_{2}\right)}{\mu_{v}^{2}\left(\beta+k_{2}+\mu_{h}\right)\left(k_{1}+\theta+\mu_{h}\right)\left(\mu_{v}+k_{3}\right)\left(\mu_{h}+\theta_{1}\right)}\right)}<1 .
$$

Thus under the condition $R_{0}<1$, the real part of all eigenvalues are negative. Hence, for $R_{0}<1$, system (1) is locally asymptotically stable. 


\subsection{At endemic equilibrium point}

We rearrange system (1) to get $S_{h}^{*}, E_{h}^{*}, I_{2 h}^{*}, R_{h}^{*}, S_{v}^{*}, E_{v}^{*}$, and $I_{v}^{*}$ in terms of $I_{1 h}^{*}$ :

$$
\left\{\begin{array}{l}
S_{h}^{*}=\frac{\Gamma_{h} k_{1}-\left(k_{1}+\theta+\mu_{h}\right)\left(\beta+k_{2}+\mu_{h}\right) I_{1 h}^{*}}{k_{1} \mu_{h}}, \\
S_{v}^{*}=\frac{\Gamma_{v}\left(\theta_{1}+\mu_{h}\right)}{\left(a c\left(\theta_{1}+\mu_{h}\right)-a d k_{2}\right) I_{1 h}^{*}+\mu_{v}\left(\theta_{1}+\mu_{h}\right)}, \\
I_{2 h}^{*}=\frac{k_{1} I_{1 h}^{*}}{\left(\theta_{1}+\mu_{h}\right)}, \quad E_{h}^{*}=\frac{\left(k_{2}+\beta+\mu_{h}\right) I_{1 h}^{*}}{k_{1}}, \\
E_{v}^{*}=\frac{\left(a c\left(\theta_{1}+\mu_{h}\right)+a d k_{2}\right) I_{h}^{*} S_{v}^{*}}{\left(\mu_{v}+k_{3}\right)\left(\theta_{1}+\mu_{h}\right)}, \quad I_{v}^{*}=\frac{k_{3} E_{v}^{*}}{\mu_{v}} \\
R_{h}^{*}=\frac{\left(\theta\left(\theta_{1}+\mu_{h}\right)\left(\beta+k_{2}+\mu_{h}\right)+\beta k_{1}\left(\theta_{1}+\mu_{h}\right)+k_{1} k_{2} \theta_{1}\right) I_{1 h}^{*}}{\mu_{h} k_{1}\left(\theta_{1}+\mu_{h}\right)} .
\end{array}\right.
$$

Theorem 3.2 If $R_{0} \in\left(1, \sqrt{\frac{\left(S_{h}^{*}+I_{h}^{*}\right)^{2}+2 a b I_{v}^{*}}{\left(\mu_{\nu}+\theta_{1}\right) S_{h}^{* 2}}}\right)$, then the endemic equilibrium $E^{*}$ of system (1) is locally asymptotically stable.

Proof. For system (1), the Jacobian matrix at $E^{*}$ is given by

$$
J^{|*|}=\left(\begin{array}{cccccccc}
-a_{11} & 0 & 0 & 0 & 0 & 0 & 0 & \frac{-2 a b\left(S_{h}^{*}\right)^{2}}{\left(S_{h}^{+*}+l_{v}^{*}\right)^{2}} \\
\frac{2 a b\left(C_{v}^{*}\right)^{2}}{\left(S_{h}^{*}++_{v}^{*}\right)^{2}} & -a_{22} & 0 & 0 & 0 & 0 & 0 & \frac{2 a b\left(S_{h}^{*}\right)^{2}}{\left(S_{h}^{*}++_{v}^{*}\right)^{2}} \\
0 & k_{1} & -\left(\beta+k_{2}+\mu_{h}\right) & 0 & 0 & 0 & 0 & 0 \\
0 & 0 & k_{2} & -\left(\theta_{1}+\mu_{h}\right) & 0 & 0 & 0 & 0 \\
0 & \theta & \beta & \theta_{1} & -\mu_{h} & 0 & 0 & 0 \\
0 & 0 & -a c S_{v}^{*} & -a d S_{v}^{*} & 0 & -a_{66} & 0 & 0 \\
0 & 0 & a c S_{v}^{*} & a d S_{v}^{*} & 0 & a_{76} & -\left(\mu_{v}+k_{2}\right) & 0 \\
0 & 0 & 0 & 0 & 0 & 0 & k_{3} & -\mu_{v}
\end{array}\right),
$$

where $a_{11}=\mu_{h}+\frac{2 a b\left(I_{v}^{*}\right)^{2}}{\left(S_{h}^{*}+I_{v}^{*}\right)^{2}}, a_{22}=\left(k_{1}+\theta+\mu_{h}\right), a_{66}=a c I_{1 h}^{*}+a d I_{2 h}^{*}+\mu_{v}$, and $a_{76}=a c I_{1 h}^{*}+$ $a d I_{2 h}^{*}$.

Clearly, one eigenvalue of the Jacobian matrix $J^{|*|}$ of model (1) around the disease present equilibrium point $E^{*}$ is negative, that is, $\lambda_{1}=-\mu_{h}<0$. For the remaining seven eigenvalues, we take the following reduced matrix:

$$
J_{1}^{|*|}=\left(\begin{array}{ccccccc}
-a_{11} & 0 & 0 & 0 & 0 & 0 & \frac{-2 a b\left(S_{h}^{*}\right)^{2}}{\left(S_{h}^{*}+I_{v}^{*}\right)^{2}} \\
\frac{2 a b\left(I_{v}^{*}\right)^{2}}{\left(S_{h}^{*}+I_{v}^{*}\right)^{2}} & -a_{22} & 0 & 0 & 0 & 0 & \frac{2 a b\left(S_{h}^{*}\right)^{2}}{\left(S_{h}^{*}+I_{v}^{*}\right)^{2}} \\
0 & k_{1} & -\left(\beta+k_{2}+\mu_{h}\right) & 0 & 0 & 0 & 0 \\
0 & 0 & k_{2} & -\left(\theta_{1}+\mu_{h}\right) & 0 & 0 & 0 \\
0 & 0 & -a c S_{v}^{*} & -a d S_{v}^{*} & -a_{66} & 0 & 0 \\
0 & 0 & a c S_{v}^{*} & a d S_{v}^{*} & a_{76} & -\left(\mu_{v}+k_{2}\right) & 0 \\
0 & 0 & 0 & 0 & 0 & k_{3} & -\mu_{v}
\end{array}\right) .
$$


After simplification we get:

$$
J_{2}^{|*|}=\left(\begin{array}{ccccccc}
-Z_{11} & 0 & 0 & 0 & 0 & 0 & -\Lambda \\
0 & -Z_{22} & 0 & 0 & 0 & 0 & \Lambda_{1} \\
0 & 0 & -Z_{33} & 0 & 0 & 0 & \Lambda_{1} k_{1} \\
0 & 0 & 0 & -Z_{44} & 0 & 0 & \Lambda_{1} k_{1} k_{2} \\
0 & 0 & 0 & 0 & -Z_{55} & -\left(\mu_{v}+k_{2}\right) & 0 \\
0 & 0 & 0 & 0 & 0 & -Z_{66} & \Lambda_{2} \\
0 & 0 & 0 & 0 & 0 & 0 & -Z_{77}
\end{array}\right)
$$

where

$$
\begin{aligned}
Z_{11}= & \left(\mu_{h}+\frac{2 a b\left(I_{v}^{*}\right)^{2}}{\left(S_{h}^{*}+I_{v}^{*}\right)^{2}}\right), Z_{22}=\left(k_{1}+\theta+\mu_{h}\right)\left(\mu_{h}+\frac{2 a b\left(I_{v}^{*}\right)^{2}}{\left(S_{h}^{*}+I_{v}^{*}\right)^{2}}\right), \\
Z_{33}= & \left(\beta+k_{2}+\mu_{h}\right)\left(k_{1}+\theta+\mu_{h}\right)\left(\mu_{h}+\frac{2 a b\left(I_{v}^{*}\right)^{2}}{\left(S_{h}^{*}+I_{v}^{*}\right)^{2}}\right), \\
Z_{44}= & \left(\beta+k_{2}+\mu_{h}\right)\left(k_{1}+\theta+\mu_{h}\right)\left(\mu_{h}+\frac{2 a b\left(I_{v}^{*}\right)^{2}}{\left(S_{h}^{*}+I_{v}^{*}\right)^{2}}\right)\left(\theta_{1}+\mu_{h}\right), \quad Z_{55}=\mu_{v}, \\
Z_{66}= & \left(\mu_{v}+k_{2}\right)\left(\beta+k_{2}+\mu_{h}\right)^{2}\left(k_{1}+\theta+\mu_{h}\right)^{2}\left(\mu_{h}+\frac{2 a b\left(I_{v}^{*}\right)^{2}}{\left(S_{h}^{*}+I_{v}^{*}\right)^{2}}\right)^{2} \\
& \times\left[a c I_{1 h}^{*}+a d I_{1 h}^{*}+\left(\mu_{v}+k_{2}\right)\right], \\
Z_{77}= & \left(k_{3}\left(\mu_{v}+k_{2}\right)\left(\beta+k_{2}+\mu_{h}\right)\left(k_{1}+\theta+\mu_{h}\right) k_{1}\left(\mu_{h}+\frac{2 a b\left(I_{v}^{*}\right)^{2}}{\left(S_{h}^{*}+I_{v}^{*}\right)^{2}}\right)\right)\left[\mu_{h} \frac{2 a c\left(I_{h}^{*}\right)^{2}}{\left(S_{v}^{*}+I_{h}^{*}\right)^{2}}\right] \\
& -\mu_{v}\left(\mu_{v}+k_{2}\right)\left(\beta+k_{2}+\mu_{h}\right)^{2}\left(k_{1}+\theta+\mu_{h}\right)^{2}\left(\mu_{h}+\frac{2 a b\left(I_{v}^{*}\right)^{2}}{\left(S_{h}^{*}+I_{v}^{*}\right)^{2}}\right)^{2} \\
& \times\left[a c I_{1 h}^{*}+a d I_{1 h}^{*}+\left(\mu_{v}+k_{2}\right)\right], \\
\Lambda= & \frac{2 a b\left(S_{h}^{*}\right)^{2}}{\left(S_{h}^{*}+I_{v}^{*}\right)^{2}}, \quad \Lambda_{1}=\frac{2 a b\left(S_{h}^{*}\right)^{2}}{\left(S_{h}^{*}+I_{v}^{*}\right)^{2}}\left(\mu_{h}+\frac{2 a b\left(I_{v}^{*}\right)^{2}}{\left(S_{h}^{*}+I_{v}^{*}\right)^{2}}\right)-\frac{2 a b\left(S_{h}^{*}\right)^{2}}{\left(S_{h}^{*}+I_{v}^{*}\right)^{2}} \frac{2 a b\left(I_{v}^{*}\right)^{2}}{\left(S_{h}^{*}+I_{v}^{*}\right)^{2}}, \\
\Lambda_{2}= & \left(\left(\mu_{v}+k_{2}\right)\left(\beta+k_{2}+\mu_{h}\right)\left(\mu_{h}+\frac{2 a b\left(I_{v}^{*}\right)^{2}}{\left(S_{h}^{*}+I_{v}^{*}\right)^{2}}\right)\left(k_{1}+\theta+\mu_{h}\right) k_{1} \frac{2 a b\left(S_{h}^{*}\right)^{2}}{\left(S_{h}^{*}+I_{v}^{*}\right)^{2}}\right. \\
& \left.\times\left(\mu_{h}+\frac{2 a b\left(I_{v}^{*}\right)^{2}}{\left(S_{h}^{*}+I_{v}^{*}\right)^{2}}+\frac{2 a b\left(I_{v}^{*}\right)^{2}}{\left(S_{h}^{*}+I_{v}^{*}\right)^{2}}\right)\right)\left[a c S_{v}^{*}+a d S_{v}^{*}\right] .
\end{aligned}
$$

The eigenvalues of $J_{2}^{|*|}$ take the following forms:

$$
\begin{aligned}
& \lambda_{2}=-\left(\mu_{h}+\frac{2 a b\left(I_{v}^{*}\right)^{2}}{\left(S_{h}^{*}+I_{v}^{*}\right)^{2}}\right)<0, \quad \lambda_{3}=-\left(k_{1}+\theta+\mu_{h}\right)\left(\mu_{h}+\frac{2 a b\left(I_{v}^{*}\right)^{2}}{\left(S_{h}^{*}+I_{v}^{*}\right)^{2}}\right)<0, \\
& \lambda_{4}=-\left(\beta+k_{2}+\mu_{h}\right)\left(k_{1}+\theta+\mu_{h}\right)\left(\mu_{h}+\frac{2 a b\left(I_{v}^{*}\right)^{2}}{\left(S_{h}^{*}+I_{v}^{*}\right)^{2}}\right)<0, \\
& \lambda_{5}=-\left(\beta+k_{2}+\mu_{h}\right)\left(k_{1}+\theta+\mu_{h}\right)\left(\mu_{h}+\frac{2 a b\left(I_{v}^{*}\right)^{2}}{\left(S_{h}^{*}+I_{v}^{*}\right)^{2}}\right)\left(\theta_{1}+\mu_{h}\right), \quad \lambda_{6}=-\mu_{v},
\end{aligned}
$$




$$
\begin{aligned}
\lambda_{7}= & -\left(\mu_{v}+k_{3}\right)\left(\beta+k_{2}+\mu_{h}\right)^{2}\left(k_{1}+\theta+\mu_{h}\right)^{2}\left(\mu_{h}+\frac{2 a b\left(I_{v}^{*}\right)^{2}}{\left(S_{h}^{*}+I_{v}^{*}\right)^{2}}\right)^{2} \\
& \times\left[a c I_{1 h}^{*}+a d I_{1 h}^{*}+\left(\mu_{v}+k_{3}\right)\right]<0,
\end{aligned}
$$

and

$$
\begin{aligned}
\lambda_{8}= & \left(k_{3}\left(\mu_{v}+k_{3}\right)\left(\beta+k_{2}+\mu_{h}\right)\left(k_{1}+\theta+\mu_{h}\right) k_{1}\left(\mu_{h}+\frac{2 a b\left(I_{v}^{*}\right)^{2}}{\left(S_{h}^{*}+I_{v}^{*}\right)^{2}}\right)\right)\left[\mu_{h} \frac{2 a c\left(I_{h}^{*}\right)^{2}}{\left(S_{v}^{*}+I_{h}^{*}\right)^{2}}\right] \\
& -\mu_{v}\left(\mu_{v}+k_{2}\right)\left(\beta+k_{2}+\mu_{h}\right)^{2}\left(k_{1}+\theta+\mu_{h}\right)^{2}\left(\mu_{h}+\frac{2 a b\left(I_{v}^{*}\right)^{2}}{\left(S_{h}^{*}+I_{v}^{*}\right)^{2}}\right)^{2} \\
& \times\left[a c I_{1 h}^{*}+a d I_{1 h}^{*}+\left(\mu_{v}+k_{3}\right)\right]<0 \\
& \text { iff } \quad 1<R_{0}<\sqrt{\left(\frac{\left(S_{h}^{*}+I_{h}^{*}\right)^{2}+2 a b I_{v}^{*}}{\left(\mu_{v}+\theta_{1}\right) S_{h}^{* 2}}\right) .}
\end{aligned}
$$

\section{Global asymptotic stability}

Here we discuss the global stability analysis of model (1) for both disease-free and endemic equilibria. We use the method of Castillo-Chavez et al. [25] to establish the global stability for disease-free equilibrium, whereas for the global stability of endemic equilibrium, we use the generalization of Lyapunov theory [26].

\subsection{At disease-free equilibrium point}

For model (1), the global stability at the disease-free point is achieved by taking into account the Castillo-Chavez approach [25]. The method is summarized by the reduction of the proposed model (1) to the following two subsystems:

$$
\begin{aligned}
& \frac{d \chi_{1}}{d t}=G\left(\chi_{1}, \chi_{2}\right), \\
& \frac{d \chi_{2}}{d t}=H\left(\chi_{1}, \chi_{2}\right) .
\end{aligned}
$$

In system (20), $\chi_{1}$ and $\chi_{2}$ represent the numbers of uninfected and infected individuals, respectively, that is, $\chi_{1}=\left(S_{h}, S_{v}, R_{h}\right) \in R^{3}$ and $\chi_{2}=\left(I_{1 h}, I_{2 h}, I_{v}, E_{h}, E_{v}\right) \in R^{4}$. The disease-free equilibrium is denoted by $E^{0}$ and defines as $E^{0}=\left(\chi_{1}^{0}, 0\right)$. Thus the existence of the global stability at the disease-free equilibrium point depends on the following two conditions:

1. If $\frac{d \chi_{1}}{d t}=G\left(\chi_{1}, 0\right)$, then $\chi_{1}^{0}$ is globally asymptotically stable.

2. $H\left(\chi_{1}, \chi_{2}\right)=B \chi_{2}-\bar{H}\left(\chi_{1}, \chi_{2}\right)$, where $\bar{H}\left(\chi_{1}, \chi_{2}\right) \geq 0$ for $\left(\chi_{1}, \chi_{2}\right) \in \Delta$.

At the second condition, $B=D_{\chi_{2}} H\left(\chi_{1}^{0}, 0\right)$ is an $M$-matrix with positive off-diagonal entries, and $\Delta$ is the feasible region.

Lemma 3 ([25]) For $R_{0}<1$, the equilibrium point $E^{0}=\left(\chi_{1}^{0}, 0\right)$ of system (1) is globally asymptotically stable if the above conditions are satisfied.

Theorem 4.1 If $R_{0}<1$, then the proposed model (1) is globally asymptotically stable at the disease-free equilibrium $E^{0}$ and unstable otherwise. 
Proof Let $\chi_{1}=\left(S_{h}, S_{v}, R_{h}\right)$ and $\chi_{2}=\left(I_{1 h}, I_{2 h}, I_{v}, E_{h}, E_{v}\right)$, and define $E^{0}=\left(\chi_{1}^{0}, 0\right)$, where

$$
\chi_{1}^{0}=\left(\frac{\Gamma_{h}}{\mu_{h}}, \frac{\Gamma_{v}}{\mu_{v}}\right) .
$$

By using model system (1) we have

$$
\begin{aligned}
& \frac{d \chi_{1}}{d t}=G\left(\chi_{1}, \chi_{2}\right), \\
& \frac{d \chi_{1}}{d t}=\left(\begin{array}{c}
\Gamma_{h}-\frac{2 a b I_{\nu}(t) S_{h}(t)}{S_{h}(t)+I_{v}(t)}-\mu_{h} S_{h}(t) \\
\theta E_{h}(t)+\beta I_{1 h}(t)+\theta_{1} I_{2 h}(t)-\mu_{h} R_{h}(t) \\
\Gamma_{v}-a c I_{1 h}(t) S_{v}(t)-a d I_{2 h}(t) S_{v}(t)-\mu_{v} S_{v}(t)
\end{array}\right) .
\end{aligned}
$$

For $S_{h}=S_{h}^{0}, S_{v}=S_{v}^{0}$, and $G\left(\chi_{1}, 0\right)=0$, we get

$$
G\left(\chi_{1}, 0\right)=\left(\begin{array}{c}
\Gamma_{h}-\mu_{h} S_{h} \\
\Gamma_{v}-\mu_{v} S_{v}
\end{array}\right)
$$

Thus from equation (23) we have that $\chi_{1} \rightarrow \chi_{1}^{0}$ as $t \rightarrow \infty$. So $\chi_{1}=\chi_{1}^{0}$ is globally asymptotically stable.

Now

$$
\begin{aligned}
& B \chi_{2}-\bar{H}\left(\chi_{1}, \chi_{2}\right) \\
& =\left(\begin{array}{ccccc}
-\left(k_{1}+\theta+\mu_{h}\right) & 0 & 0 & 0 & 2 a b \\
k_{1} & -\left(\beta+k_{2}+\mu_{h}\right) & 0 & 0 & 0 \\
0 & k_{2} & -\left(\mu_{h}+\theta_{1}\right) & 0 & 0 \\
0 & a c S_{v}^{0} & a d S_{v}^{0} & -\left(\mu_{v}+k_{3}\right) & 0 \\
0 & 0 & 0 & k_{3} & -\mu_{v}
\end{array}\right)\left(\begin{array}{c}
E_{h} \\
I_{1 h} \\
I_{2 h} \\
E_{v} \\
I_{v}
\end{array}\right) \\
& -\left(\begin{array}{c}
2 a b-\frac{2 a b I_{v} S_{h}}{S_{h}+I_{v}} \\
0 \\
0 \\
a c S_{v}^{0}+a d S_{v}^{0}-\left(a c I_{1 h} S_{v}+a d I_{2 h} S_{v}\right) \\
0
\end{array}\right) .
\end{aligned}
$$

As the total population is bounded by $S_{h}^{0}$ and $S_{v}^{0}$, that is $S_{h} \leq S_{h}^{0}$ and $S_{v} \leq S_{v}^{0}$, so $2 a b \geq$ $\frac{2 a b I_{v} S_{h}}{S_{h}+I_{v}}$ and $a c S_{v}^{0}+a c S_{v}^{0} \geq\left(a c I_{1 h} S_{v}+a d I_{2 h} S_{v}\right)$, which implies that $\bar{H}\left(\chi_{1}, \chi_{2}\right) \geq 0$. Clearly, $B$ is an $M$-matrix, and hence both the conditions are proved, so by Lemma 1 the disease-free equilibrium point $E^{0}$ is stable globally asymptotically.

\subsection{Endemic equilibrium (global stability)}

"For the global stability of (1) at the endemic equilibrium $E^{*}$, we use the geometrical approach [26]. Thus we investigate the sufficient condition through which the $E^{*}$ is globally asymptotically stable. Therefore consider the differential equation

$$
\dot{x}=f(x),
$$


where the open set $U \subset R^{n}$ is simply connected, and $f: U \rightarrow R^{n}$ is a function such that $f \in$ $C^{1}(U)$. Assuming that $f\left(x^{*}\right)=0$ is the solution of equation (25), for $x\left(t, x_{0}\right)$, the following are true:

3. There exist a compact absorbing set $K \in U$.

4. System (25) has a unique equilibrium.

The solution $x^{*}$ is said to be globally asymptotically stable in $U$ if it is locally asymptotically stable and all trajectories in $U$ converge to the equilibrium $x^{*}$. For $n \geq 2$, a condition satisfied for $f$, which precludes the existence of nonconstant periodic solution of equation (25), is known as the Bendixson criterion. The classical Bendixson criterion $\operatorname{div} f(x)<0$ for $n=2$ is robust under $C^{1}$ [26]. Furthermore, a point $x_{0} \in U$ is wandering for equation (25) if there exist a neighborhood $N$ of $x_{0}$ and $\tau>0$ such that $N \cap x(t, N)$ is empty for all $t>\tau$. Thus the following global stability principle established for autonomous system in any finite dimension.

Lemma 4 If the conditions (3)-(4) and Bendixson criterion are satisfied for equation (25) (i.e., robust under $C^{1}$ local perturbation off at all nonequilibrium nonwandering point for equation (25)), then $x^{*}$ is globally asymptotically stable in $U$, provided that it is stable.

Define a matrix-valued function $P$ on $U$ by

$$
P(x)=\left(\begin{array}{l}
n \\
1
\end{array}\right) \times\left(\begin{array}{l}
n \\
1
\end{array}\right)
$$

Further, assume that $P^{-1}$ exists and is continuous for $x \in K$. Now define the quantity

$$
\bar{q}=\limsup _{t \rightarrow \infty} \frac{1}{t} \int_{0}^{t}\left[\mu\left(B\left(x\left(s, x_{0}\right)\right)\right)\right] d s,
$$

where $J^{[2]}$ is the second additive compound matrix of $J$, that is, $J(x)=U f(x)$ and $B=P_{f} P^{-1}+$ $P{ }^{[2]} P^{-1}$. Let $\ell(B)$ be the Lozinski measure of the matrix $B$ with respect to the norm $\|\cdot\|$ in $R^{n}$ [27] defined by

$$
\ell(B)=\lim _{x \rightarrow 0} \frac{|I+B x|-1}{x} .
$$

Hence if $\bar{q}<0$, then the presence of any orbit gives rise to a simple closed rectifiable curve such as a periodic orbit and heterocyclic cycle.

Lemma 5 Let $U$ be simply connected, and let conditions (3)-(4) be satisfied. Then the unique equilibrium $x^{*}$ of equation (25) is globally asymptotically stable in $U$ if $\bar{q}<0$.

Now we apply the above techniques to prove the global stability of model (1) at the endemic equilibrium. We have the following stability.

Theorem 4.2 If $\frac{2 b\left(I_{v}^{*}\right)^{2}}{\left(S_{h}^{*}+I_{v}^{*}\right)^{2}}>\frac{E_{h}\left(c I_{h}^{*}+d I_{2 h}^{*}\right)}{S_{h}},\left(c I_{1 h}^{*}+d I_{2 h}^{*}\right)>\frac{2 b E_{\nu}\left(I_{v}^{*}\right)^{2}}{S_{\nu}\left(S_{h}^{*}+I_{v}^{*}\right)^{2}}$, and $R_{0}>1$, then model (29) is globally asymptotically stable at the endemic equilibrium $E^{*}$ and unstable otherwise. 
Proof To prove the global asymptotic stability of the proposed model (1) at the endemic equilibrium $E^{*}$, let us consider the subsystem of (1)

$$
\left\{\begin{array}{l}
\frac{d S_{h}}{d t}=\Gamma_{h}-a b\left(\frac{2 I_{v}(t) S_{h}(t)}{S_{h}(t)+I_{v}(t)}\right)-\mu_{h} S_{h}(t), \\
\frac{d E_{h}}{d t}=a b\left(\frac{2 I_{v}(t) S_{h}(t)}{S_{h}(t)+I_{\nu}(t)}\right)-\left(k_{1}+\theta+\mu_{h}\right) E_{h}(t), \\
\frac{d S_{v}}{d t}=\Gamma_{v}-a c I_{1 h}(t) S_{v}(t)-a d I_{2 h}(t) S_{v}(t)-\mu_{v} S_{v}(t), \\
\frac{d E_{v}}{d t}=a c I_{1 h}(t) S_{v}(t)+a d I_{2 h}(t) S_{v}(t)-\left(\mu_{v}+k_{3}\right) E_{v}(t) .
\end{array}\right.
$$

Let us start with the Jacobian matrix of system (29)

$$
J=\left(\begin{array}{llll}
j_{11} & j_{12} & j_{13} & j_{14} \\
j_{21} & j_{22} & j_{23} & j_{24} \\
j_{31} & j_{32} & j_{33} & j_{34} \\
j_{41} & j_{42} & j_{43} & j_{44}
\end{array}\right)
$$

The third additive compound matrix is given by

$$
J^{|3|}=\left(\begin{array}{cccc}
j_{11}+j_{22}+j_{33} & j_{34} & -j_{24} & j_{14} \\
j_{43} & j_{11}+j_{22}+j_{44} & j_{23} & -j_{13} \\
-j_{42} & j_{32} & j_{11}+j 33+j_{44} & j_{12} \\
j_{41} & -j_{31} & j_{21} & j_{22}+j_{33}+j_{44}
\end{array}\right) .
$$

Let $J$ be the Jacobian matrix of system (29) given by

$$
J=\left(\begin{array}{cccc}
-\left(\mu_{h}+\frac{2 a b\left(I_{*}^{*}\right)^{2}}{\left(S_{h}^{*}+I_{v}^{*}\right)^{2}}\right. & 0 & 0 & 0 \\
\frac{2 a b\left(I_{v}^{*}\right)^{2}}{\left(S_{h}^{*}+I_{v}^{*}\right)^{2}} & -\left(k_{1}+\theta+\mu_{h}\right) & 0 & 0 \\
0 & 0 & -\left(\mu_{v}+a c I_{1 h}^{*}+a d I_{2 h}^{*}\right) & 0 \\
0 & 0 & \left(a c I_{1 h}^{*}+a d I_{2 h}^{*}\right) & -\left(\mu_{v}+k_{3}\right)
\end{array}\right) .
$$

The third additive compound matrix of $J$ is denoted by $J^{|3|}$ and defined as

$$
J^{|3|}=\left(\begin{array}{cccc}
A_{11} & 0 & 0 & 0 \\
\left(a c I_{1 h}^{*}+a d I_{2 h}^{*}\right) & A_{22} & 0 & 0 \\
0 & 0 & A_{33} & 0 \\
0 & 0 & \frac{2 a b\left(I_{v}^{*}\right)^{2}}{\left(S_{h}^{*}+I_{v}^{*}\right)^{2}} & A_{44}
\end{array}\right)
$$

where

$$
\begin{aligned}
A_{11} & =j_{11}+j_{22}+j_{33} \\
& =-\left(\frac{2 a b\left(I_{v}^{*}\right)^{2}}{\left(S_{h}^{*}+I_{v}^{*}\right)^{2}}+k_{1}+\theta+2 \mu_{h}+\mu_{v}+a c I_{1 h}^{*}+a d I_{2 h}^{*}\right), \\
A_{22} & =j_{11}+j_{22}+j_{44} \\
& =-\left(\frac{2 a b\left(I_{v}^{*}\right)^{2}}{\left(S_{h}^{*}+I_{v}^{*}\right)^{2}}+\mu_{v}+k_{3}+k_{1}+\theta+2 \mu_{h}\right),
\end{aligned}
$$




$$
\begin{aligned}
A_{33} & =j_{11}+j_{33}+j_{44} \\
& =-\left(\frac{2 a b\left(I_{v}^{*}\right)^{2}}{\left(S_{h}^{*}+I_{v}^{*}\right)^{2}}+\mu_{h}+a c I_{1 h}^{*}+a d I_{2 h}^{*}+2 \mu_{v}+k_{3}\right), \\
A_{44} & =j_{22}+j_{33}+j_{44} \\
& =-\left(k+\theta+\mu_{h}+a c I_{1 h}^{*}+a d I_{2 h}^{*}+2 \mu_{v}+k_{3}\right) .
\end{aligned}
$$

Let us choose a function $p(\chi)=p\left(S_{h}, E_{h}, S_{v}, E_{v}\right)$ so that $p(\chi)=\operatorname{diag}\left\{S_{h}, E_{h}, S_{v}, E_{v}\right\}$, which implies that $p^{-1}(\chi)=\operatorname{diag}\left\{\frac{1}{S_{h}}, \frac{1}{E_{h}}, \frac{1}{S_{v}}, \frac{1}{E_{v}}\right\}$. The time derivative of $p(\chi)$ yields that $P_{f}(\chi)=$ $\operatorname{diag}\left\{\dot{S}_{h}, \dot{E}_{h}, \dot{S}_{v}, \dot{E}_{v}\right\}$.

A direct computation shows that $B=P_{f} P^{-1}+P J^{|3|} P^{-1}$, which becomes

$$
\begin{aligned}
& P_{f} P^{-1}=\operatorname{diag}\left\{\frac{\dot{S_{h}}}{S_{v}}, \frac{\dot{E_{h}}}{E_{h}}, \frac{\dot{S_{v}}}{S_{v}}, \frac{\dot{E}_{v}}{E_{v}}\right\}, \\
& P J^{|3|} P^{-1}=\left(\begin{array}{cccc}
A_{11} & 0 & 0 & 0 \\
\frac{E_{h}}{S_{h}}\left(a c I_{1 h}^{*}+a d I_{2 h}^{*}\right) & A_{22} & 0 & 0 \\
0 & 0 & A_{33} & 0 \\
0 & 0 & \frac{2 a b\left(I_{v}^{*}\right)^{2}}{\left(S_{h}^{*}+I_{v}^{*}\right)^{2}} \frac{E_{v}}{S_{v}} & A_{44}
\end{array}\right),
\end{aligned}
$$

so that $B=P_{f} P^{-1}+P J^{|3|} P^{-1}$,

$$
B=\left(\begin{array}{cccc}
a_{11} & 0 & 0 & 0 \\
a_{21} & a_{22} & 0 & 0 \\
0 & 0 & a_{33} & 0 \\
0 & 0 & a_{43} & a_{44}
\end{array}\right),
$$

where

$$
\begin{aligned}
& a_{11}=\frac{\dot{S}_{h}}{S_{h}}-\left(\frac{2 a b\left(I_{v}^{*}\right)^{2}}{\left(S_{h}^{*}+I_{v}^{*}\right)^{2}}+k_{1}+\theta+2 \mu_{h}+\mu_{v}+a c I_{1 h}^{*}+a d I_{2 h}^{*}\right), \\
& a_{21}=\frac{E_{h}}{S_{h}}\left(a c I_{1 h}^{*}+a d I_{2 h}^{*}\right), \quad a_{22}=\frac{\dot{E}_{h}}{E_{h}}-\left(\frac{2 a b\left(I_{v}^{*}\right)^{2}}{\left(S_{h}^{*}+I_{v}^{*}\right)^{2}}+\mu_{v}+k_{3}+k_{1}+\theta+2 \mu_{h}\right), \\
& a_{43}=\frac{2 a b\left(I_{v}^{*}\right)^{2}}{\left(S_{h}^{*}+I_{v}^{*}\right)^{2}} \frac{E_{v}}{S_{v}}, \quad a_{33}=\frac{\dot{S}_{v}}{S_{v}}-\left(\frac{2 a b\left(I_{v}^{*}\right)^{2}}{\left(S_{h}^{*}+I_{v}^{*}\right)^{2}}+\mu_{h}+a c I_{1 h}^{*}+a d I_{2 h}^{*}+2 \mu_{v}+k_{3}\right), \\
& a_{44}=\frac{\dot{E}_{v}}{E_{v}}-\left(k_{!}+\theta+\mu_{h}+a c I_{1 h}^{*}+a d I_{2 h}^{*}+2 \mu_{v}+k_{3}\right) .
\end{aligned}
$$

Consequently,

$$
\begin{aligned}
h_{1}(t) & =a_{11}+\sum_{j=2}^{4}\left|a_{1 j}\right|, \\
h_{1}(t) & =\frac{\dot{S}_{h}}{S_{h}}-\left(\frac{2 a b\left(I_{v}^{*}\right)^{2}}{\left(S_{h}^{*}+I_{v}^{*}\right)^{2}}+k_{1}+\theta+2 \mu_{h}+\mu_{v}+a c I_{1 h}^{*}+a d I_{2 h}^{*}\right) \\
& \leq \frac{\dot{S_{h}}}{S_{h}}-\left(2 \mu_{h}+k_{1}+\theta+\mu_{v}\right),
\end{aligned}
$$


and if $\frac{2 b\left(I_{V}^{*}\right)^{2}}{\left(S_{h}^{*}+I_{v}^{*}\right)^{2}}>\frac{E_{h}\left(c I_{1 h}^{*}+d I_{2 h}^{*}\right)}{S_{h}}$, then

$$
\begin{aligned}
h_{2}(t) & =a_{22}+\sum_{j=1 \text { and } j \neq 2}^{4}\left|a_{2 j}\right|, \\
h_{2}(t) & =\frac{\dot{E_{h}}}{E_{h}}-\left(\mu_{v}+k_{3}+k_{1}+\theta+2 \mu_{h}\right)-\frac{2 a b\left(I_{v}^{*}\right)^{2}}{\left(S_{h}^{*}+I_{v}^{*}\right)^{2}}+\frac{E_{h}}{S_{h}}\left(a c I_{1 h}^{*}+a d I_{2 h}^{*}\right) \\
& =\frac{\dot{E_{h}}}{E_{h}}-\left(\mu_{v}+k_{3}+k_{1}+\theta+2 \mu_{h}\right)-a\left(\frac{2 b\left(I_{v}^{*}\right)^{2}}{\left(S_{h}^{*}+I_{v}^{*}\right)^{2}}-\frac{E_{h}\left(c I_{1 h}^{*}+d I_{2 h}^{*}\right)}{S_{h}}\right) \\
& \leq \frac{\dot{E_{h}}}{E_{h}}-\left(\mu_{v}+k_{3}+k_{1}+\theta+2 \mu_{h}\right),
\end{aligned}
$$

Similarly,

$$
\begin{aligned}
h_{3}(t) & =a_{33}+\sum_{j=1 \text { and } j \neq 3}^{4}\left|a_{3 j}\right|, \\
h_{3}(t) & =\frac{\dot{S}_{v}}{S_{v}}-\left(\frac{2 a b\left(I_{v}^{*}\right)^{2}}{\left(S_{h}^{*}+I_{v}^{*}\right)^{2}}+\mu_{h}+a c I_{1 h}^{*}+a d I_{2 h}^{*}+2 \mu_{v}+k_{3}\right) \\
& \leq \frac{\dot{S}_{v}}{S_{v}}-\left(\mu_{h}+2 \mu_{v}+k_{3}\right)
\end{aligned}
$$

and if $\left(c I_{1 h}^{*}+d I_{2 h}^{*}\right)>\frac{2 b E_{v}\left(I_{V}^{*}\right)^{2}}{S_{v}\left(S_{h}^{*}+I_{v}^{*}\right)^{2}}$, then

$$
\begin{aligned}
h_{4}(t) & =a_{44}+\sum_{j=1}^{3}\left|a_{4 j}\right|, \\
h_{4}(t) & =\frac{\dot{E}_{v}}{E_{v}}-\left(k_{1}+\theta+\mu_{h}+2 \mu_{v}+k_{3}\right)-\left(a c I_{1 h}^{*}+a d I_{2 h}^{*}\right)+\frac{2 a b\left(I_{v}^{*}\right)^{2}}{\left(S_{h}^{*}+I_{v}^{*}\right)^{2}} \frac{E_{v}}{S_{v}} \\
& =\frac{\dot{E}_{v}}{E_{v}}-\left(k_{1}+\theta+\mu_{h}+2 \mu_{v}+k_{3}\right)-a\left(\left(c I_{1 h}^{*}+d I_{2 h}^{*}\right)-\frac{2 b E_{v}\left(I_{v}^{*}\right)^{2}}{S_{v}\left(S_{h}^{*}+I_{v}^{*}\right)^{2}}\right) \\
& \leq \frac{\dot{E}_{v}}{E_{v}}-\left(k_{1}+\theta+\mu_{h}+2 \mu_{v}+k_{3}\right) .
\end{aligned}
$$

Let $\left(b_{1}, b_{2}, b_{3}, b_{4}\right)$ be a vector in $\mathbf{R}^{4}$. The Lozinski measure $\mu(B)$ is defined as $\mu(B)=h_{i}$, $i=1,2,3,4$. The integration of the Lozinski measure $\mu(B)$ and taking the limits as $t \rightarrow \infty$ lead to the following equations:

$$
\begin{aligned}
\limsup _{t \rightarrow \infty} \frac{1}{t} \int_{0}^{t} h_{1}(t) d t & \leq \frac{1}{t} \log \frac{S_{h}(t)}{S_{h}(0)}-\left(2 \mu_{h}+k_{1}+\theta+\mu_{v}\right) \\
& <-\left(2 \mu_{h}+k_{1}+\theta+\mu_{v}\right), \\
\limsup _{t \rightarrow \infty} \frac{1}{t} \int_{0}^{t} h_{2}(t) d t & \leq \frac{1}{t} \log \frac{E_{h}(t)}{E_{h}(0)}-\left(\mu_{h}+\mu_{v}+k_{3}+k_{1}+\theta+\mu_{h}\right) \\
& <-\left(2 \mu_{h}+\mu_{v}+k_{3}+k_{1}+\theta\right),
\end{aligned}
$$




$$
\begin{aligned}
\limsup _{t \rightarrow \infty} \frac{1}{t} \int_{0}^{t} h_{3}(t) d t & \leq \frac{1}{t} \log \frac{S_{v}(t)}{S_{v}(0)}-\left(\mu_{h}+2 \mu_{v}+k_{3}\right) \\
& <-\left(\mu_{h}+2 \mu_{v}+k_{3}\right), \\
\limsup _{t \rightarrow \infty} \frac{1}{t} \int_{0}^{t} h_{4}(t) d t & \leq \frac{1}{t} \log \frac{E_{v}(t)}{E_{v}(0)}-\left(k_{1}+\theta+\mu_{h}+2 \mu_{v}+k_{3}\right) \\
& <-\left(k_{1}+\theta+\mu_{h}+2 \mu_{v}+k_{3}\right) .
\end{aligned}
$$

Thus combining these four inequalities, we get the following equation:

$$
\bar{q}=\limsup _{t \rightarrow \infty} \frac{1}{t} \int_{0}^{t} \mu(B) d t<0 .
$$

The system containing the first four equations of model (1) is globally asymptotically stable around its interior equilibrium $\left(S_{h}^{*}, E_{h}^{*}, S_{v}^{*}, E_{v}^{*}\right)$. Now consider the subsystem of model (1)

$$
\left\{\begin{array}{l}
\frac{d I_{1 h}}{d t}=k_{1} E_{h}(t)-\left(\beta+\mu_{h}+k_{2}\right) I_{1 h}(t) \\
\frac{d I_{2 h}}{d t}=k_{2} I_{1 h}(t)-\left(\mu_{h}+\theta_{1}\right) I_{2 h}(t) \\
\frac{d R_{h}}{d t}=\theta E_{h}(t)+\beta I_{1 h}(t)+\theta_{1} I_{2 h}(t)-\mu_{h} R_{h}(t) \\
\frac{d I_{v}}{d t}=k_{3} E_{v}(t)-\mu_{v} I_{v}(t)
\end{array}\right.
$$

Now rewrite the system in the form

$$
\left\{\begin{array}{l}
\frac{d I_{1 h}}{d t}+\left(\beta+\mu_{h}+k_{2}\right) I_{1 h}(t)=k_{1} E_{h}^{*}(t) \\
\frac{d I_{2 h}}{d t}+\left(\mu_{h}+\theta_{1}\right) I_{2 h}(t)=k_{2} I_{1 h}^{*}(t) \\
\frac{d R_{h}}{d t}+\mu_{h} R_{h}(t)=\theta E_{h}^{*}(t)+\beta I_{1 h}^{*}(t)+\theta_{1} I_{2 h}^{*}(t) \\
\frac{d I_{v}}{d t}+\mu_{v} I_{v}(t)=k_{3} E_{v}^{*}(t)
\end{array}\right.
$$

The integrating factors for the system are $e^{t\left(\beta+k_{2}+\mu_{h}\right)}, e^{t\left(\mu_{h}+\theta_{1}\right)}, e^{t\left(\mu_{h}\right)}$, and $e^{t\left(\mu_{v}\right)}$.

Using the integrating factors, we solve the system. So for large time $t$, that is, $t \rightarrow \infty$, $I_{1 h} \rightarrow I_{1 h}^{*}, I_{2 h} \rightarrow I_{2 h}^{*}, R_{h} \rightarrow R_{h}^{*}$, and $I_{v} \rightarrow I_{v}^{*}$, which means that the endemic equilibrium point $E^{*}$ is globally asymptotically stable.

\section{Sensitivity analysis}

Determining the parameters that are helpful in decreasing the spread of infectious disease is carried out by sensitivity analysis. Forward sensitivity analysis is considered a vital component of disease modeling although its computation becomes tedious for complex biological models. Sensitivity analysis of $R_{0}$ has received much attention from the ecologists and epidemiologists. The behavior of the $R_{0}$ with some suitable values of the parameters are shown in Fig. 1.

Definition 1 The normalized forward sensitivity index of $R_{0}$ that depends differentiably on a parameter $\omega$ is defined as

$$
S_{\omega}=\frac{\omega}{R_{0}} \frac{\partial R_{0}}{\partial \omega} .
$$




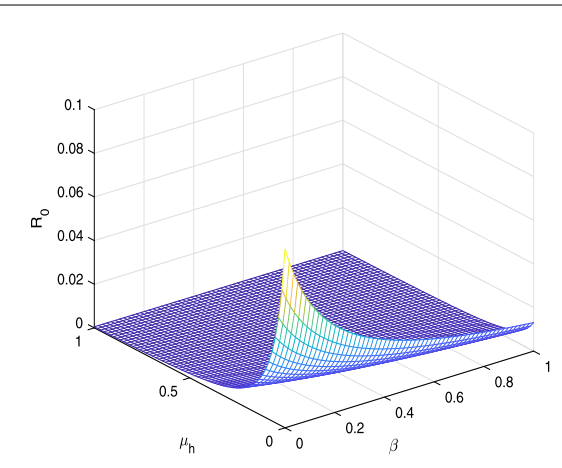

(a) $R_{0}$ versus sensitive parameters $\mu_{h}$ and $\beta$.

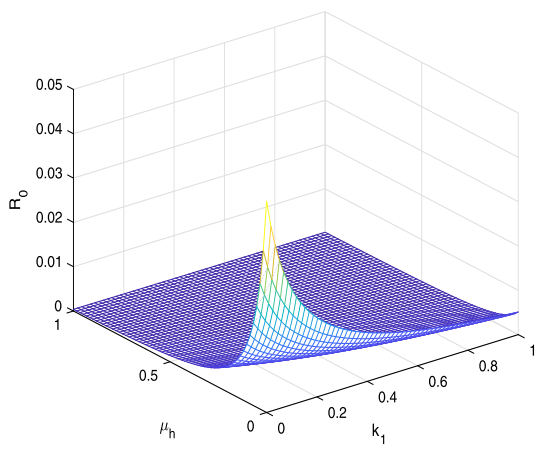

(c) $R_{0}$ versus sensitive parameters $\mu_{h}$ and $k_{1}$.

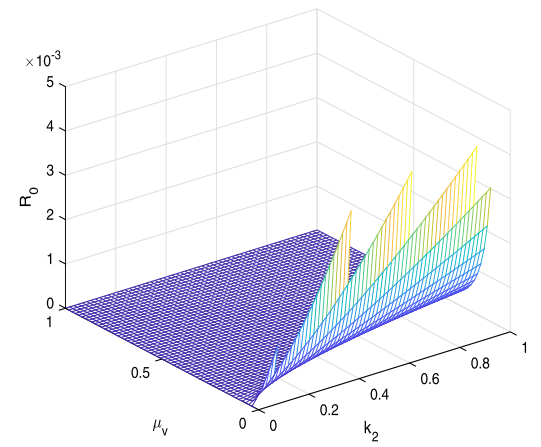

(e) $R_{0}$ versus sensitive parameters $\mu_{v}$ and $k_{2}$.

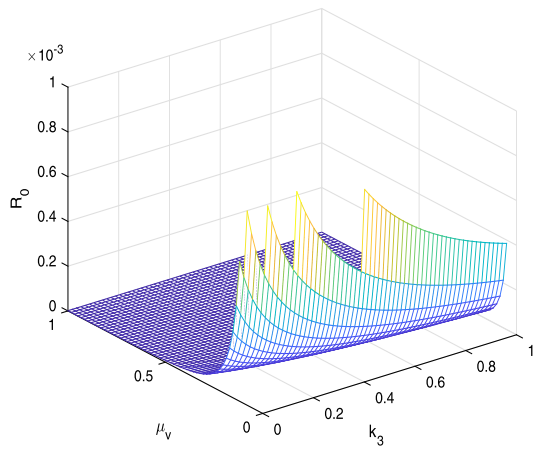

(g) $R_{0}$ versus sensitive parameters $\mu_{v}$ and $k_{3}$.

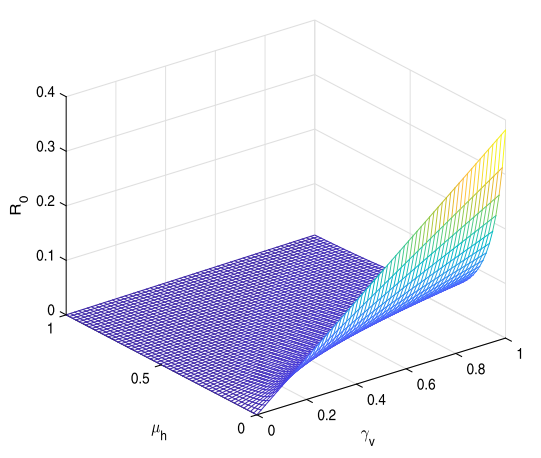

(b) $R_{0}$ versus sensitive parameters $\mu_{h}$ and $\gamma_{v}$.

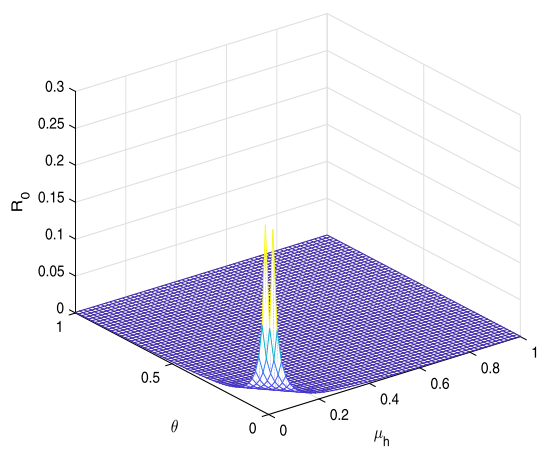

(d) $R_{0}$ versus sensitive parameters $\mu_{h}$ and $\theta$.

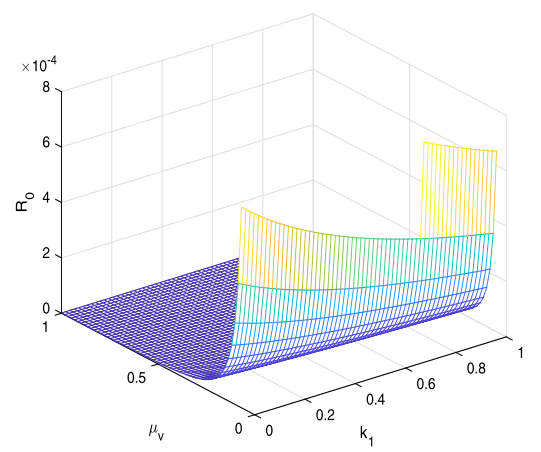

(f) $R_{0}$ versus sensitive parameters $\mu_{v}$ and $k_{1}$.

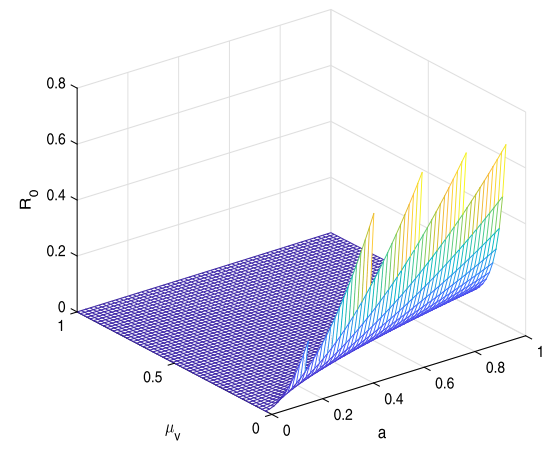

(h) $R_{0}$ versus sensitive parameters $\mu_{v}$ and $a$.

Figure 1 Sensitivity analysis of different parameters 
Table 1 Sensitivity indices of the reproduction number $R_{0}$ against mentioned parameters

\begin{tabular}{lll}
\hline Parameter & S. Index & Value \\
\hline$a$ & $S_{a}$ & 1.000000000 \\
$\theta$ & $S_{\theta}$ & -0.004792332271 \\
$c$ & $S_{C}$ & 0.5000000001 \\
$\mu_{h}$ & $S_{\mu_{h}}$ & -0.3685739184 \\
$\beta$ & $S_{\beta}$ & -0.2272727272 \\
$\Gamma_{V}$ & $S_{\Gamma_{V}}$ & 0.0 \\
$d$ & $S_{d}$ & 1.000000000 \\
\hline
\end{tabular}

\begin{tabular}{lll}
\hline Parameter & S. Index & Value \\
\hline$b$ & $S_{b}$ & 0.4999999999 \\
$k_{1}$ & $S_{k_{1}}$ & 0.1006389778 \\
$k_{2}$ & $S_{k_{2}}$ & 0.01754385961 \\
$\mu_{v}$ & $S_{\mu_{V}}$ & -0.5175438600 \\
$\Gamma_{h}$ & $S_{\Gamma_{h}}$ & 0.0 \\
$\theta_{1}$ & $S_{\theta_{1}}$ & 0.0254635451 \\
$k_{3}$ & $S_{k_{3}}$ & 0.4999999999 \\
\hline
\end{tabular}

Three methods are normally used to calculate the sensitivity indices: (i) by direct differentiation, (ii) by a Latin hypercube sampling method, and (iii) by linearizing system (1) and then solving the obtained set of linear algebraic equations. We will apply the direct differentiation method as it gives analytical expressions for the indices. The indices not only show us the influence of various aspects associated with the spreading of infectious disease but also gives us important information regarding the comparative change between $R_{0}$ and different parameters. Consequently, it helps in developing the control strategies. Table 1 shows that the parameters $a, b, k_{1}, c, k_{2}, \theta_{1}, d$, and $k_{3}$ have a positive influence on the reproduction number $R_{0}$; for example, the growth or decay of these parameters, say, by $10 \%$ will increase or decrease the reproduction number by $10 \%, 4.9 \%, 1.0 \%, 5.0 \%$, $0.17 \%, 0.25 \%, 10 \%$, and $4.9 \%$, respectively. On the other hand, the indices for parameters $\theta, \mu_{h}, \mu_{v}$, and $\beta$ illustrate that increasing their values by $10 \%$ will decrease the values of reproduction number $R_{0}$ by $0.04 \%, 3.6 \%, 5.1 \%$, and $2.2 \%$, respectively. Note that $\Gamma_{h}$ and $\Gamma_{v}$ have no impact on the reproduction number.

\section{Optimal control}

By using these control variables our control problem becomes

$$
\left\{\begin{aligned}
\frac{d S_{h}}{d t}= & \Gamma_{h}-\frac{2\left(1-u_{1}(t)\right) a b I_{v}(t) S_{h}(t)}{S_{h}(t) I_{v}(t)}-\mu_{h} S_{h}(t), \\
\frac{d E_{h}}{d t}= & \frac{2\left(1-u_{1}(t)\right) a b I_{v}(t) S_{h}(t)}{S_{h}(t)+I_{v}(t)}-\left(k_{1}+\theta+\mu_{h}\right) E_{h}(t), \\
\frac{d I_{1 h}}{d t}= & k_{1} E_{h}(t)-\left(u_{2}(t)+\beta+\mu_{h}+k_{2}\right) I_{1 h}(t), \\
\frac{d I_{2 h}}{d t}= & k_{2} I_{1 h}(t)-\left(u_{2}(t)+\mu_{h}+\theta_{1}\right) I_{2 h}(t), \\
\frac{d R_{h}}{d t}= & \theta E_{h}(t)+\left(\beta+u_{2}(t)\right) I_{1 h}(t)+\left(\theta_{1}+u_{2}(t)\right) I_{2 h}(t)-\mu_{h} R_{h}(t), \\
\frac{d S_{v}}{d t}= & \Gamma_{v}-\left(1-u_{1}(t)\right) a c I_{1 h}(t) S_{v}(t)-\left(1-u_{1}(t)\right) a d I_{2 h}(t) S_{v}(t) \\
& -\left(u_{3}(t)+\mu_{v}\right) S_{v}(t), \\
\frac{d E_{v}}{d t}= & \left(1-u_{1}(t)\right) a c I_{1 h}(t) S_{v}(t)+\left(1-u_{1}(t)\right) a d I_{2 h}(t) S_{v}(t) \\
& -\left(u_{3}(t)+\mu_{v}+k_{3}\right) E_{v}(t), \\
\frac{d I_{v}}{d t}= & k_{3} E_{v}(t)-\left(u_{3}(t)+\mu_{v}\right) I_{v}(t) .
\end{aligned}\right.
$$

The goal of our optimal control strategies is minimizing the infectious and exposed human population, the vector population, sandfly biting rate, and the cost of implementing the control by using possible minimal control variables $u_{1}(t), u_{2}(t)$, and $u_{3}(t)$. To do this, we 
use the bounded Lebesgue-measurable control to construct the objective functional

$$
\begin{aligned}
J\left(u_{1}, u_{2}, u_{3}\right)= & \int_{0}^{1}\left(g_{1} E_{h}(t)+g_{2} I_{1 h}(t)+g_{3} I_{2 h}(t)+g_{4}\left(S_{v}(t)+E_{v}(t)+I_{v}(t)\right)\right. \\
& \left.+\frac{1}{2}\left(d_{1} u_{1}^{2}(t)+d_{2} u_{2}^{2}(t)+d_{3} u_{3}^{2}(t)\right)\right) d t
\end{aligned}
$$

subject to system (37).

In the objective functional, $g_{1}, g_{2}, g_{3}$, and $g_{4}$ represent the weight constants of the exposed, infectious human, and of vector population, respectively, $d_{1}, d_{2}$, and $d_{3}$ are weight constants of human self-protection, human treatment, and vector control, respectively. The terms $(1 / 2) d_{1} u_{1}^{2}(t),(1 / 2) d_{2} u_{2}^{2}(t)$, and $(1 / 2) d_{3} u_{3}^{2}(t)$ describe the costs of disease interventions. The cost associated with the first control strategy $u_{1}(t)$ comes from the cost of sandfly repellent lotions, electric mats, and mosquito bed nets. The cost associated with the second control strategy $u_{2}(t)$ is the cost of expensive medication of human class. The cost associated with the third control strategy $u_{3}(t)$ can arise from applying different types chemical pesticides to kill sand flies at any stage of their life. We have assumed the costs as proportional to the square of the corresponding control function. We aim to find control functions such that

$$
J\left(u_{1}^{*}, u_{2}^{*}, u_{3}^{*}\right)=\min \left\{J\left(u_{1}, u_{2}, u_{3}\right),\left(u_{1}, u_{2}, u_{3}\right) \in D\right\}
$$

subject to system (4). The control set $D$ is defined as

$$
D=\left\{\left(u_{1}, u_{2}, u_{3}\right) \mid u_{i}(t) \text { is Lebesgue measurable on }[0,1], 0 \leq u_{i}(t)<1, i=1,2,3\right\} .
$$

\subsection{Existence of the control problem}

For the existence of the control problem, we consider the control system (37) with initial conditions at $t=0$. In case of bounded Lebesgue-measurable controls and nonnegative initial conditions, there exists a nonnegative bounded solution of the state system [28, 29]. For optimal solution of the system, we first find the Lagrangian and Hamiltonian. We define the Lagrangian of the control problem (37) as

$$
L(t)=g_{1} E_{h}(t)+g_{2} I_{1 h}(t)+g_{3} I_{2 h}(t)+g_{4} N_{v}(t)+\frac{1}{2}\left(d_{1} u_{1}^{2}(t)+d_{2} u_{2}^{2}(t)+d_{3} u_{3}^{2}(t)\right),
$$

where $N_{v}(t)=S_{v}(t)+E_{v}(t)+I_{v}(t)$.

We define the Hamiltonian $H$ to find the minimal value of the Lagrangian as follows:

$$
\begin{aligned}
H(t)= & g_{1} E_{h}(t)+g_{2} I_{1 h}(t)+g_{3} I_{2 h}(t)+g_{4} N_{v}(t)+\frac{1}{2}\left[d_{1} u_{1}^{2}(t)+d_{2} u_{2}^{2}(t)+d_{3} u_{3}^{2}(t)\right] \\
& +\left[\lambda_{1}(t)\left(\Gamma_{h}-\frac{2\left(1-u_{1}(t)\right) a b I_{v}(t) S_{h}(t)}{S_{h}(t)+I_{v}(t)}-\mu_{h} S_{h}(t)\right)\right] \\
& +\left[\lambda_{2}(t)\left(\frac{2\left(1-u_{1}(t)\right) a b I_{v}(t) S_{h}(t)}{S_{h}(t)+I_{v}(t)}-\left(k_{1}+\theta+\mu_{h}\right) E_{h}(t)\right)\right] \\
& +\left[\lambda_{3}(t)\left(k_{1} E_{h}(t)-\left(u_{2}(t)+\beta+\mu_{h}+k_{2}\right) I_{1 h}(t)\right)\right] \\
& +\left[\lambda_{4}(t)\left(k_{2} I_{1 h}(t)-\left(u_{2}(t)+\mu_{h}+\theta_{1}\right) I_{2 h}(t)\right)\right] \\
& +\left[\lambda_{5}(t)\left(\theta E_{h}(t)+\left(\beta+u_{2}(t)\right) I_{1 h}(t)+\left(\theta_{1}+u_{2}(t)\right) I_{2 h}(t)-\mu_{h} R_{h}(t)\right)\right]
\end{aligned}
$$




$$
\begin{aligned}
& +\left[\lambda _ { 6 } ( t ) \left(\Gamma_{v}-\left(1-u_{1}(t)\right) a c I_{1 h}(t) S_{v}(t)-\left(1-u_{1}(t)\right) a d I_{2 h}(t) S_{v}(t)\right.\right. \\
& \left.\left.-\left(u_{3}(t)+\mu_{v}\right) S_{v}(t)\right)\right] \\
& +\left[\lambda _ { 7 } ( t ) \left(\left(1-u_{1}(t)\right) a c I_{1 h}(t) S_{v}(t)+\left(1-u_{1}(t)\right) a d I_{2 h}(t) S_{v}(t)\right.\right. \\
& \left.\left.-\left(u_{3}(t)+\mu_{v}+k_{3}\right) E_{v}(t)\right)\right] \\
& +\left[\lambda_{8}(t)\left(k_{3} E_{v}(t)-\left(u_{3}(t)+\mu_{v}\right) I_{v}(t)\right)\right] .
\end{aligned}
$$

Theorem 6.1 There exists an optimal control $u^{*}=\left(u_{1}^{*}, u_{2}^{*}, u_{3}^{*}\right) \in D$ such that $J\left(u_{1}, u_{2}, u_{3}\right)=$ $\min _{\left(u_{1}, u_{2}, u_{3}\right) \in D} J\left(u_{1}, u_{2}, u_{3}\right)$ subject to system (37) and initial conditions at $t=0$.

Proof The control variables and the state variables are nonnegative. So we use the result in $[28,29]$ for the existence of an optimal control. The necessary convexity of the objective functional in $u_{1}, u_{2}$, and $u_{3}$ is satisfied here. Also, by definition the set of the control variables $\left(u_{1}, u_{2}, u_{3}\right) \in D$ is convex and closed. The compactness, which we need for the existence of an optimal control, is confirmed by the boundedness of the optimal system. Also, the integrand in the functional (38), $g_{1} E_{h}(t)+g_{2} I_{1 h}(t)+g_{3} I_{2 h}(t)+g_{4} N_{v}(t)+\frac{1}{2}\left(d_{1} u_{1}^{2}(t)+\right.$ $\left.d_{2} u_{2}^{2}(t)+d_{3} u_{3}^{2}(t)\right)$, is convex on the control set $D$. We can find a constant $\eta>1$ and positive numbers $\xi_{1}$ and $\xi_{2}$ such that $J\left(u_{1}, u_{2}, u_{3}\right) \geq \xi_{1}\left(\left|u_{1}\right|^{2},\left|u_{2}\right|^{2},\left|u_{3}\right|^{2}\right)^{\frac{\eta}{2}}-\xi_{2}$, as the state variables are bounded. This completes the proof of the existence of an optimal control.

For optimal solution, we apply Pontryagin's maximum principle [30] as follows. If $(x(t), u(t))$ is an optimal solution of an optimal control problem, then there exists a nontrivial vector function $\lambda(t)=\left(\lambda_{1}(t), \lambda_{2}(t), \ldots, \lambda_{n}(t)\right)$ satisfying the following:

$$
\begin{aligned}
& \frac{d x(t)}{d t}=\frac{\partial H(t, x, u, \lambda)}{\partial \lambda}, \\
& 0=\frac{\partial H(t, x, u, \lambda)}{\partial u}, \\
& \lambda^{\prime}(t)=-\frac{\partial H(t, x, u, \lambda)}{\partial x} .
\end{aligned}
$$

We apply the necessary conditions to the Hamiltonian $H$ in system (40).

Theorem 6.2 Let $S_{h}^{*}, E_{h}^{*}, I_{h}^{*}, R_{h}^{*}, S_{v}^{*}, E_{v}^{*}$, and $I_{v}^{*}$ be optimal state solutions with associated optimal control variables $\left(u_{1}^{*}, u_{2}^{*}, u_{3}^{*}\right)$ for the optimal control problem system (37)-(38). Then there exist adjoint variables $\lambda_{i}, i=1,2, \ldots, 8$, satisfying

$$
\left\{\begin{aligned}
\frac{d \lambda_{1}(t)}{d t}= & \lambda_{1}(t) \frac{2\left(1-u_{1}(t)\right) a b I_{v}^{* 2}(t)}{\left(S_{h}^{*}(t)+I_{v}^{*}(t)\right)^{2}}-\lambda_{2}(t) \frac{2\left(1-u_{1}(t)\right) a b I_{v}^{* 2}(t)}{\left(S_{h}^{*}(t)+I_{v}^{*}(t)\right)^{2}}-\lambda_{1}(t) \mu_{h}, \\
\frac{d \lambda_{2}(t)}{d t}= & \lambda_{2}(t)\left(k_{1}+\theta+\mu_{h}\right)-\lambda_{3}(t) k_{1}-\lambda_{5}(t) \theta-g_{1}, \\
\frac{d \lambda_{3}(t)}{d t}= & \left(\lambda_{6}-\lambda_{7}\right)\left(1-u_{1}(t)\right) a c S_{v}^{*}-\lambda_{4} k_{2}+\lambda_{3}\left(u_{2}(t)+\beta+\mu_{h}+k_{2}\right) \\
& -\lambda_{5}\left(\beta+u_{2}(t)\right)-g_{2}, \\
\frac{d \lambda_{4}(t)}{d t}= & \left(\lambda_{6}-\lambda_{7}\right)\left(1-u_{1}(t)\right) a d S_{v}^{*}+\lambda_{4}\left(u_{2}(t)+\mu_{h}+\theta_{1}\right)-\lambda_{5}\left(\theta_{1}+u_{2}(t)\right)-g_{3}, \\
\frac{d \lambda_{5}(t)}{d t}= & \lambda_{5} \mu_{h}, \\
\frac{d \lambda_{6}(t)}{d t}= & \left(\lambda_{6}-\lambda_{7}\right)\left(1-u_{1}(t)\right) a c I_{1 h}^{*}(t)+\left(\lambda_{6}-\lambda_{7}\right)\left(1-u_{1}(t)\right) a d I_{2 h}^{*}(t) \\
& +\lambda_{6}\left(u_{3}(t)+\mu_{v}\right)-g_{4}, \\
\frac{d \lambda_{7}(t)}{d t}= & \lambda_{7}\left(u_{3}(t)+\mu_{v}+k_{3}\right)-\lambda_{8} k_{3}-g_{3}, \\
\frac{d \lambda_{8}(t)}{d t}= & \lambda_{1}(t) \frac{2\left(1-u_{1}(t)\right) a b S_{h}^{* 2}(t)}{\left(S_{h}^{*}(t)+I_{v}^{*}(t)\right)^{2}}-\lambda_{2}(t) \frac{2\left(1-u_{1}(t)\right) a b S_{h}^{* 2}(t)}{\left(S_{h}^{*}(t)+I_{v}^{*}(t)\right)^{2}}+\left(u_{3}(t)+\mu_{v}\right) \lambda_{8}-g_{4} .
\end{aligned}\right.
$$


We have the transversality conditions

$$
\lambda_{i}(T)=0, \quad i=1,2,3, \ldots, 8,
$$

for the control set $u_{i}$, and hence we have

$$
\frac{\partial H}{\partial u_{i}}=0, \quad i=1,2,3
$$

By the optimality condition we have

$$
\left.\frac{\partial H}{\partial u_{1}}\right|_{u_{1}=u_{1}^{*}}=0
$$

that is,

$$
\frac{\partial H}{\partial u_{1}}=d_{1} u_{1}+\left(\lambda_{1}-\lambda_{2}\right) \frac{2 a b I_{v}^{*}(t) S_{h}^{*}(t)}{\left(S_{h}^{*}(t)+I_{v}^{*}(t)\right)}+\left(\lambda_{6}-\lambda_{7}\right) a c I_{1 h}^{*}(t) S_{v}^{*}+\left(\lambda_{6}-\lambda_{7}\right) a d I_{2 h}^{*}(t) S_{v}^{*}
$$

Similarly,

$$
\begin{aligned}
\left.\frac{\partial H}{\partial u_{1}}\right|_{u_{1}=u_{1}^{*}}= & d_{1} u_{1}^{*}+\left(\lambda_{1}-\lambda_{2}\right) \frac{2 a b I_{v}^{*}(t) S_{h}^{*}(t)}{\left(S_{h}^{*}(t)+I_{v}^{*}(t)\right)}+\left(\lambda_{6}-\lambda_{7}\right) a c I_{1 h}^{*}(t) S_{v}^{*} \\
& +\left(\lambda_{6}-\lambda_{7}\right) a d I_{2 h}^{*}(t) S_{v}^{*}=0,
\end{aligned}
$$

or

$$
u_{1}^{*}=\frac{\left(\lambda_{2}-\lambda_{1}\right) \frac{2 a b I_{v}^{*}(t) S_{h}^{*}(t)}{\left(S_{h}^{*}(t)+I_{v}^{*}(t)\right)}+\left(\lambda_{7}-\lambda_{6}\right) a c I_{1 h}^{*}(t) S_{v}^{*}+\left(\lambda_{7}-\lambda_{6}\right) a d I_{2 h}^{*}(t) S_{v}^{*}}{d_{1}} .
$$

Also,

$$
\left.\frac{\partial H}{\partial u_{2}}\right|_{u_{2}=u_{2}^{*}}=0
$$

that is,

$$
\frac{\partial H}{\partial u_{2}}=d_{2} u_{2}^{*}+\left(\lambda_{5}-\lambda_{3} I_{1 h}^{*}\right)+\left(\lambda_{5}-\lambda_{4} I_{2 h}^{*}\right)=0,
$$

or

$$
u_{2}^{*}=\frac{\left(\lambda_{3}-\lambda_{5} I_{1 h}^{*}\right)+\left(\lambda_{4}-\lambda_{5} I_{2 h}^{*}\right)}{d_{2}},
$$

and

$$
\left.\frac{\partial H}{\partial u_{3}}\right|_{u_{3}=u_{3}^{*}}=0,
$$


that is,

$$
\frac{\partial H}{\partial u_{3}}=d_{3} u_{3}^{*}-\lambda_{7} E_{v}^{*}(t)-\lambda_{8} I_{v}^{*}(t)=0
$$

or

$$
u_{3}^{*}=\frac{\lambda_{7} E_{v}^{*}(t)+\lambda_{8} I_{v}^{*}(t)}{d_{3}} .
$$

Now using an appropriate variation argument and taking the bounds into account, we obtain

$$
\begin{aligned}
u_{1}^{*}(t)= & \max \left\{\min \left\{\frac{\left(\lambda_{2}-\lambda_{1}\right) \frac{2 a b I_{v}^{*}(t) S_{h}^{*}(t)}{\left(S_{h}^{*}(t)+I_{v}^{*}(t)\right)}+\left(\lambda_{7}-\lambda_{6}\right) a c I_{1 h}^{*}(t) S_{v}^{*}+\left(\lambda_{7}-\lambda_{6}\right) a d I_{2 h}^{*}(t) S_{v}^{*}}{d_{1}}, 1\right\},\right. \\
& 0\}, \\
u_{2}^{*}(t)= & \left.\max \left\{\min \left\{\frac{\left(\lambda_{3}-\lambda_{5} I_{1 h}^{*}\right)+\left(\lambda_{4}-\lambda_{5} I_{2 h}^{*}\right.}{d_{2}}\right), 1\right\}, 0\right\}, \\
u_{3}^{*}(t)= & \max \left\{\min \left\{\frac{\lambda_{7} E_{v}^{*}(t)+\lambda_{8} I_{v}^{*}(t)}{d_{3}}, 1\right\}, 0\right\} .
\end{aligned}
$$

These are optimal, as required.

Proof To find adjoint equations and the transversality conditions, we use Eq. (37). Differentiating the Hamiltonian $H$ with respect to each state variable, we obtain system (41). Solving the equations $\frac{\partial H}{\partial u_{1}}=0, \frac{\partial H}{\partial u_{2}}=0$, and $\frac{\partial H}{\partial u_{3}}=0$ on the interior of the control set and using the optimality conditions and the property of the control space $D$, we derive Eqs. (43). The optimal control and the state are found by solving the optimality system (38), the adjoint system $\frac{d \lambda_{i}}{d t}$, initial and boundary conditions (42), and equations of the optimal control (43). Since the second derivatives of the Lagrangian with respect to $u_{1}, u_{2}$, and $u_{3}$ are positive, the optimal problem is minimum at the controls $u_{1}^{*}, u_{2}^{*}$, and $u_{3}^{*}$. By substituting the values of $u_{1}^{*}, u_{2}^{*}$, and $u_{3}^{*}$ into the control system (40) we get the following system:

$$
\left\{\begin{aligned}
\frac{d S_{h}^{*}}{d t}= & \Gamma_{h}-\frac{2\left(1-u_{1}^{*}(t)\right) a b I_{I^{*}}^{*}(t) S_{h}^{*}(t)}{S_{h}^{*}(t) I_{v}}-\mu_{h} S_{h}^{*}(t), \\
\frac{d E_{h}^{*}}{d t}= & \frac{2\left(1-u_{1}^{*}(t)\right) a b I_{h}^{*}(t) S_{h}^{*}(t)}{S_{h}^{*}(t)+I_{v}^{*}(t)}-\left(k_{1}+\theta+\mu_{h}\right) E_{h}^{*}(t), \\
\frac{d I_{1 h}^{*}}{d t}= & k_{1} E_{h}^{*}(t)-\left(u_{2}^{*}(t)+\beta+\mu_{h}+k_{2}\right) I_{1 h}^{*}(t), \\
\frac{d I_{2 h}^{*}}{d t}= & k_{2} I_{1 h}^{*}(t)-\left(u_{2}^{*}(t)+\mu_{h}+\theta_{1}\right) I_{2 h}^{*}(t), \\
\frac{d R_{h}^{*}}{d t}= & \theta E_{h}^{*}(t)+\left(\beta+u_{2}^{*}(t)\right) I_{1 h}^{*}(t)+\left(\theta_{1}+u_{2}^{*}(t)\right) I_{2 h}^{*}(t)-\mu_{h} R_{h}^{*}(t), \\
\frac{d S_{v}^{*}}{d t}= & \Gamma_{v}-\left(1-u_{1}^{*}(t)\right) a c I_{1 h}^{*}(t) S_{v}^{*}(t)-\left(1-u_{1}^{*}(t)\right) a d I_{2 h}(t) S_{v}^{*}(t) \\
& -\left(u_{3}^{*}(t)+\mu_{v}\right) S_{v}^{*}(t), \\
\frac{d E_{v}^{*}=}{d t}= & \left(1-u_{1}^{*}(t)\right) a c I_{1 h}^{*}(t) S_{v}^{*}(t)+\left(1-u_{1}^{*}(t)\right) a d I_{2 h}^{*}(t) S_{v}^{*}(t) \\
& -\left(u_{3}^{*}(t)+\mu_{v}+k_{3}\right) E_{v}^{*}(t), \\
\frac{d I_{v}^{*}}{d t}= & k_{3} E_{v}^{*}(t)-\left(u_{3}^{*}(t)+\mu_{v}\right) I_{v}^{*}(t),
\end{aligned}\right.
$$




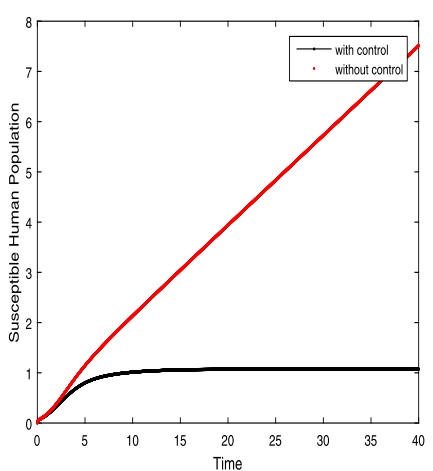

(a) The plot represents the susceptible individuals.

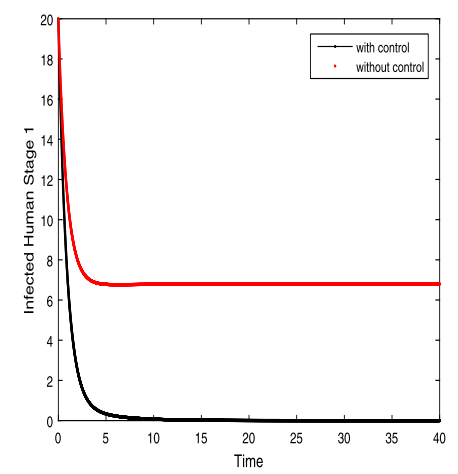

(c) The plot represents the infected individuals.

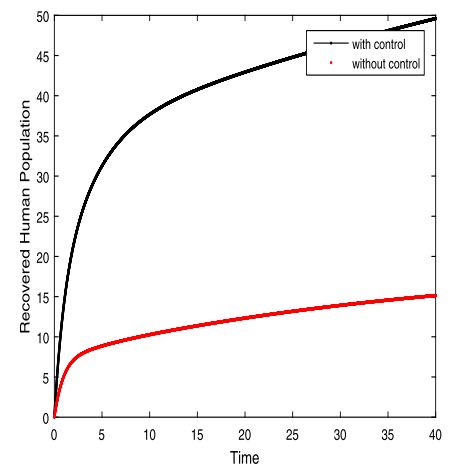

(e) The plot represents the exposed vectors.

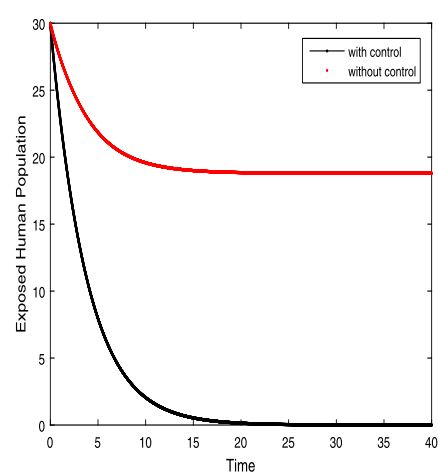

(b) The plot represents the exposed individuals.

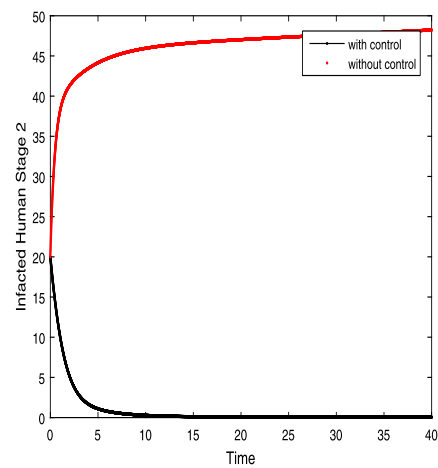

(d) The plot represents the infected individuals of stage 2 .

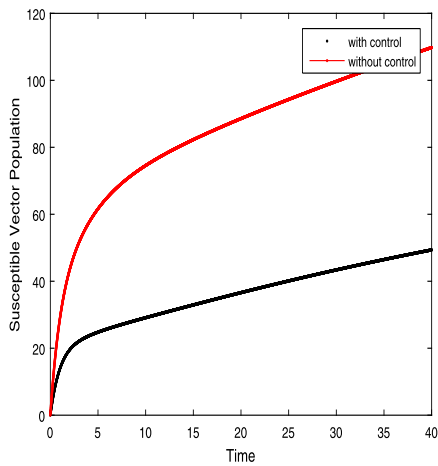

(f) The plot represents the susceptible vectors.

Figure 2 Plots of different classes showing the difference between "with control" and "without control" 


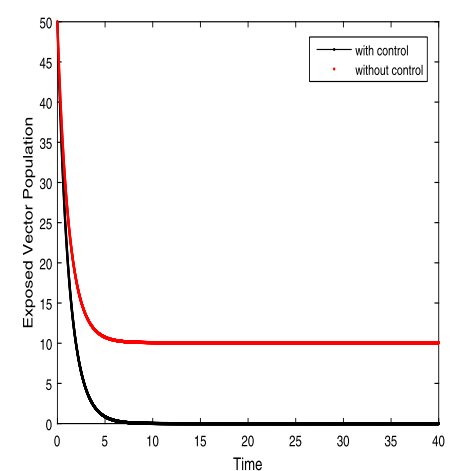

(g) The plot represents the exposed vectors.

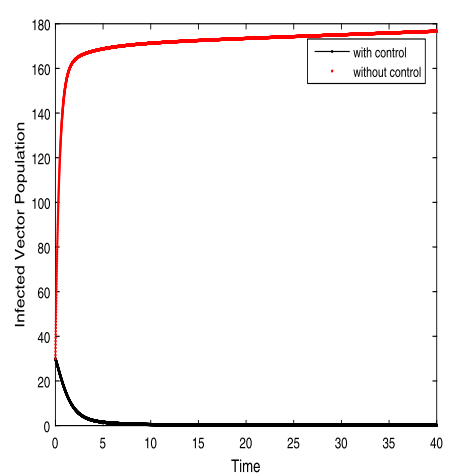

(h) The plot represents the infected vectors.

Figure 2 Continued

with

$$
\begin{aligned}
H^{*}(t)= & g_{1} E_{h}^{*}(t)+g_{2} I_{1 h}^{*}(t)+g_{3} I_{2 h}^{*}(t)+g_{4} N_{v}^{*}(t)+\frac{1}{2}\left[d_{1} u_{1^{2}}^{*}(t)+d_{2} u_{2^{2}}^{*}(t)+d_{3} u_{3^{2}}^{*}(t)\right] \\
& +\left[\lambda_{1}(t)\left(\Gamma_{h}-\frac{2\left(1-u_{1}(t)\right) a b I_{v}^{*}(t) S_{h}^{*}(t)}{S_{h}^{*}(t)+I_{v}^{*}(t)}-\mu_{h} S_{h}^{*}(t)\right)\right] \\
& +\left[\lambda_{2}(t)\left(\frac{2\left(1-u_{1}^{*}(t)\right) a b I_{v}^{*}(t) S_{h}^{*}(t)}{S_{h}^{*}(t)+I_{v}^{*}(t)}-\left(k_{1}+\theta+\mu_{h}\right) E_{h}^{*}(t)\right)\right] \\
& +\left[\lambda_{3}(t)\left(k_{1} E_{h}^{*}(t)-\left(u_{2}^{*}(t)+\beta+\mu_{h}+k_{2}\right) I_{1 h}^{*}(t)\right)\right] \\
& +\left[\lambda_{4}(t)\left(k_{2} I_{1 h}^{*}(t)-\left(u_{2}^{*}(t)+\mu_{h}+\theta_{1}\right) I_{2 h}^{*}(t)\right)\right] \\
& +\left[\lambda_{5}(t)\left(\theta E_{h}^{*}(t)+\left(\beta+u_{2}^{*}(t)\right) I_{1 h}^{*}(t)+\left(\theta_{1}+u_{2}^{*}(t)\right) I_{2 h}^{*}(t)-\mu_{h} R_{h}^{*}(t)\right)\right] \\
& +\left[\lambda _ { 6 } ( t ) \left(\Gamma_{v}-\left(1-u_{1}^{*}(t)\right) a c I_{1 h}^{*}(t) S_{v}^{*}(t)-\left(1-u_{1}^{*}(t)\right) a d I_{2 h}^{*}(t) S_{v}^{*}(t)\right.\right. \\
& \left.\left.-\left(u_{3}(t)+\mu_{v}\right) S_{v}^{*}(t)\right)\right] \\
& +\left[\lambda _ { 7 } ( t ) \left(\left(1-u_{1}^{*}(t)\right) a c I_{1 h}(t) S_{v}^{*}(t)+\left(1-u_{1}^{*}(t)\right) a d I_{2 h}^{*}(t) S_{v}^{*}(t)\right.\right. \\
& \left.\left.-\left(u_{3}^{*}(t)+\mu_{v}+k_{3}\right) E_{v}^{*}(t)\right)\right] \\
& +\left[\lambda_{8}(t)\left(k_{3} E_{v}^{*}(t)-\left(u_{3}^{*}(t)+\mu_{v}\right) I_{v}^{*}(t)\right)\right] .
\end{aligned}
$$

The profile of each of the state variables with and without control have been depicted in Fig. 2.

\section{Conclusions}

We study a five-grade Anthroponotic Cutaneous Leishmania epidemic model with harmonic mean type incidence rate. For endemic stability analysis, we consider a generalization of the Lyapunov method, called the geometrical approach, in which a second additive compound matrix is taken into account. The feasibility of our result is verified by numerical simulations. Extending our work, we can use a harmonic mean type incidence rate to reformulate a visceral model for the leishmaniasis epidemic. We can also check its stability analysis, parameter sensitivity, bifurcation analysis, and optimal control. It is strongly 
recommended that proper record keeping and documentation systems for leishmaniasis be initiated by health authorities at the local, provincial, and national levels and be well maintained to identify leishmaniasis outbreaks so that control measures can be started well in time. Further, IDP camps must be monitored regularly to minimize the risk that nonendemic areas will be exposed to the disease by infected IDPs.

\section{Acknowledgements}

The authors acknowledge with thanks the support of the Deanship of Scientific Research (DSR) at King Abdulaziz University, Jeddah.

\section{Funding}

Not applicable.

Availability of data and materials

Not applicable.

\section{Competing interests}

The authors declare that they have no competing interests.

\section{Authors' contributions}

All authors contributed equally to the manuscript and typed, read, and approved the final manuscript.

\section{Author details}

${ }^{1}$ Department of Physics, University of Malakand, Chakdara Dir, Khyber Pakhtunkhwa, Pakistan. ${ }^{2}$ Department of Basic Sciences, University of Engineering and Technology, Peshawar, Khyber Pakhtunkhwa, Pakistan. ${ }^{3}$ Department of Mathematics and Statistics, University of Swat, Swat, Khyber Pakhtunkhawa, Pakistan. ${ }^{4}$ Department of Computer Engineering, Biruni University, Istanbul, Turkey. ${ }^{5}$ Department of Mathematics, Federal University Dutse, Jigawa, Nigeria. ${ }^{6}$ Department of Mathematics, Faculty of Science, King Abdulaziz University, Jeddah 21589, Saudi Arabia.

\section{Publisher's Note}

Springer Nature remains neutral with regard to jurisdictional claims in published maps and institutional affiliations.

Received: 16 December 2020 Accepted: 18 January 2021 Published online: 28 January 2021

\section{References}

1. Hussain, M., Munir, S., Jamal, M.A., Ayaz, S., Akhoundi, M., Mohamed, K.: Epidemic outbreak of anthroponotic cutaneous leishmaniasis in Kohat District, Khyber Pakhtunkhwa, Pakistan. Acta Trop. 172, 147-155 (2017)

2. Sacks, D.L., Perkins, P.V.: Development of infective stage leishmania promastigotes within phlebotomine sandflies. Am. J. Trop. Med. Hyg. 34, 456-467 (1985)

3. Harre, J., Dorsey, K., Armstrong, L., Burge, J., Kinnamon, K.: Comparative fecundity and survival rates of Phlebotomus papatasi sandflies membrane fed on blood from eight mammal species. Med. Vet. Entomol. 15(2), 189-196 (2001)

4. Kasap, O.E., Alten, B.: Comparative demography of the sand fly Phlebotomus papatasi (Diptera: Psychodidae) at constant temperatures. J. Vector Ecol. 31(2), 378-385 (2006)

5. Porrozzi, R., Teva, A., Amara, V.F., Santos da Costa, M.V., Grimaldi, G.J.R.: Cross-immunity experiments between different species or strains of leishmania in rhesus macaques (Macaca mulatta). Am. J. Trop. Med. Hyg. 17(3), 297-305 (2004)

6. Kolaczinski, J., Brooker, S., Reyburn, H., Rowland, M.: Epidemiology of anthroponotic cutaneous leishmaniasis in Afghan refugee camps in northwest Pakistan. Trans. R. Soc. Trop. Med. Hyg. 98, 373-378 (2004)

7. Chaves, L.F., Hernandez, M.J.: Mathematical modelling of American cutaneous leishmaniasis: incidental hosts and threshold conditions for infection persistence. Acta Trop. 92, 245-252 (2004)

8. Chaves, L.F., Cohen, M.J., Pascual, M., Wilson, M.L.: Social exclusion modfies climate and deforestation impacts on a vector-borne disease. PLoS Negl. Trop. Dis. 2(2), e176 (2008)

9. Das, P., Mukherjee, D., Sarkar, A.K.: Effect of delay on the model of American cutaneous leishmaniasis. J. Biol. Syst. 15(2), 139-147 (2007)

10. Chaves, L.F.: Climate and recruitment limitation of hosts: the dynamics of American cutaneous leishmaniasis seen through semi-mechanistic seasonal models. Ann. Trop. Med. Parasitol. 103, 221-234 (2009)

11. Bacaer, N., Guernaoui, S.: The epidemic threshold of vector-borne diseases with seasonality the case of cutaneous leishmaniasis in Chichaoua, Morocco. J. Math. Biol. 53, 421-436 (2006)

12. Calzada, J.E., Saldaña, A., Rigg, C., Valderrama, A., Romero, L., Chaves, L.F.: Changes in phlebotomine sandfly species composition following insecticide thermal fogging in a rural setting of western Panama. PLoS ONE 8(1), e53289 (2013)

13. Akgül, E.K.: Solutions of the linear and nonlinear differential equations within the generalized fractional derivatives. Chaos 29, 023108 (2019). https://doi.org/10.1063/1.5084035

14. Atangana, A., Akgül, A.: On solutions of fractal fractional differential equations. Discrete Contin. Dyn. Syst., Ser. S (2020). https://doi.org/10.3934/dcdss.2020421

15. Akgül, A.: A novel method for a fractional derivative with non-local and non-singular kernel. Chaos Solitons Fractals $114,478-482(2018)$ 
16. Atangana, A., Akgül, A.: Can transfer function and Bode diagram be obtained from Sumudu transform. Alex. Eng. J. 59(4), 1971-1984 (2020)

17. Owolbi, K.M., Atangana, A., Akgül, A.: Modelling and analysis of fractal-fractional partial differential equations: application to reaction-diffusion model. Alex. Eng. J. 59(4), 2477-2490 (2020)

18. Atangana, A., Akgül, A., Owolbi, K.M.: Analysis of fractal fractional differential equations. Alex. Eng. J. 59(3), 1117-1134 (2020)

19. Abdon, A., Iğret Araz, S.: Nonlinear equations with global differential and integral operators: existence, uniqueness with application to epidemiology. Results Phys. 20, 103593 (2021)

20. Atangana, A., lğret Araz, S.: Mathematical model of COVID-19 spread in Turkey and South Africa: theory, methods, and applications. Adv. Differ. Equ. 2020, 659 (2020). https://doi.org/10.1186/s13662-020-03095

21. İğret Araz, S.: Analysis of a Covid-19 model: optimal control, stability and simulations. Alex. Eng. J. 60(1), 647-658 (2021)

22. Khan, A., Zarin, R., Inc, M., et al.: Stability analysis of leishmania epidemic model with harmonic mean type incidence rate. Eur. Phys. J. Plus 135, 528 (2020). https://doi.org/10.1140/epjp/s13360-020-00535-0

23. Van den Driessche, P., Watmough, J.: Reproduction number and sub-threshold endemic equilbria for compartmental models of disease transmission. Math. Biosci. 180, 29-38 (2002)

24. Khan, A., Zarin, R., Hussain, G., Ahmad, N.A., Mohd, M.H., Yusuf, A.: Stability analysis and optimal control of COVID-19 with convex incidence rate in Khyber Pakhtunkhawa (Pakistan). Results Phys. (2020). https://doi.org/10.1016/.j.rinp.2020.103703

25. Castillo-Chavez, C., Feng, Z., Huang, W.: Mathematical approaches for emerging and reemerging infectious diseases: an introduction. In: Proceedings of the IMA, vol. 125, pp. 229-250. Springer, Berlin (2002)

26. Li, M.Y., Muldowney, J.S.: A geometric approach to global-stability problems. SIAM J. Math. Anal. 27(4), 1070-1083 (2006)

27. Martin, R.H.: Logarithmic norms and projections applied to linear differential systems. J. Math. Anal. Appl. 45(2), 432-454 (1974)

28. Zaman, G., Kang, Y.H., Jung, I.H.: Stability analysis and optimal vaccination of an SIR epidemic model. Biosystems 93 240-249 (2008). https://doi.org/10.1016/j.biosystems.2008.05.004PMID:18584947

29. Birkhoff, G., Rota, G.C.: Ordinary Differential Equations, 4th edn. Wiley, New York (1989)

30. Kamien, M.I., Schwartz, N.L.: Dynamics Optimization: The Calculus of Variations and Optimal Control in Economics and Management (1991)

\section{Submit your manuscript to a SpringerOpen ${ }^{0}$ journal and benefit from:}

- Convenient online submission

- Rigorous peer review

- Open access: articles freely available online

- High visibility within the field

- Retaining the copyright to your article

Submit your next manuscript at $\boldsymbol{~ s p r i n g e r o p e n . c o m ~}$ 